\title{
Technology-Based Assessments and Treatments of Anxiety in Autistic Individuals: Systematic Review and Narrative Synthesis
}

\author{
Lucy Adams $^{1} \mathbb{D} \cdot$ Lucia Valmaggia $^{1} \cdot$ Emily Simonoff $^{1}$
}

Received: 12 January 2021 / Accepted: 10 June 2021 / Published online: 1 July 2021

(c) The Author(s) 2021

\begin{abstract}
This systematic review (Prospero Registration Number: CRD42019142910) aimed to narratively synthesise technology-aided assessments and treatments of anxiety in individuals with autism spectrum disorder (ASD) for the first time. Sixteen studies were identified: 5 assessment studies and 11 treatment studies. Assessment studies targeted state anxiety using ecological momentary assessment, wearables, or computerised tasks. Treatment studies targeted specific fears/phobias using electronic screen media or transdiagnostic anxiety using telemedicine. Broadly, results indicated technology-aided assessments and treatments may be feasible and effective at targeting anxiety in ASD, except treatments involving social scripts or peer modelling. Assessment results further indicated that state anxiety in ASD has a distinct psychophysiological signature and is evoked by idiosyncratic triggers. However, larger scale studies with representative samples are needed.
\end{abstract}

Keywords Autism spectrum disorder $\cdot$ Anxiety $\cdot$ Ecological momentary assessment $\cdot$ Wearables $\cdot$ Electronic screen media . Telemedicine

Autism spectrum disorder (ASD) is a lifelong neurodevelopmental disorder characterised by impairments in social interaction and communication, as well as the presence of repetitive and restricted behaviours/interests/activities (American Psychiatric Association, 2013). Anxiety disorders commonly co-occur with ASD (Hossain et al., 2020), and there are ASD-specific challenges in their assessment and treatment, so the interface between anxiety and ASD is a research priority - a view shared by autistic ${ }^{1}$ individuals and their parents (Wallace et al., 2013). A fundamental challenge is that anxiety symptoms in ASD can be atypical in how they are triggered, their presentation, and their conceptual overlap with ASD symptomatology (e.g. Lau et al., 2020; Magiati et al., 2017). Atypical anxiety triggers associated with ASD include idiosyncratic specific fears, disruptions to routine and change, social confusion, sensory overload, and being reoriented away from restricted or repetitive patterns of behaviour, interests, or activities. Atypical presentations of anxiety associated with ASD include increased sensory,

Lucy Adams

Lucy.1.adams@kcl.ac.uk

1 Institute of Psychiatry, Psychology \& Neuroscience, King's College London, 16 De Crespigny Park, London SE5 8AF, UK repetitive, ritualistic, or socially inappropriate behaviours. Accompanying challenges that complicate the assessment and treatment of anxiety include ASD-associated impairments in social communication, emotional literacy, interoceptive abilities, and recollecting and narrating past experiences (Bordignon et al., 2015; Kinnaird et al., 2019; Marini et al., 2018; Palser et al., 2018), alongside the heterogeneity that characterises the autistic population. To address these ASD-specific challenges, assessment and treatments for anxiety need adapting. Digital technology may aid adaptations as it can be used to provide novel approaches that can ease personalisation. These approaches involve the use of electronic devices and software, such as apps, computerand internet-based approaches, wearable devices, and virtual reality (VR), in adjunct or independent to mental health services (Hollis et al., 2018; see definitions in Table 1).

Digital technology may specifically aid the assessment of anxiety in ASD since it can facilitate the use of methods that are multi-modal (e.g. clinical interviews, rating scales, behavioural observations, and physiological measurements) and from multiple informants (e.g. parents, therapist, and self-report), which is recommended to reduce the reliance

\footnotetext{
1 The term "autistic" will be used herein for consistency and because there is a general preference in the autistic community for this term (Kenny et al., 2016).
} 
Table 1 Types and definitions of digital technologies

\begin{tabular}{|c|c|}
\hline Term & Description used \\
\hline Digital technology & $\begin{array}{l}\text { Informed by Hollis et al.'s (2018) definition, technologies that the participant does not directly inter- } \\
\text { act with are not included, with the exception of technologies passively monitoring physiological } \\
\text { signals. This definition thus encompasses electronic devices and software such as apps, comput- } \\
\text { ers, websites, videoconferencing, mobile platforms, wearable devices, VR, augmented reality, and } \\
\text { robots }\end{array}$ \\
\hline VR & $\begin{array}{l}\text { Three-dimensional computer-generated visual environments displayed on a screen of an electronic } \\
\text { device (e.g. a head-mounted display). The images are typically synchronised to the movements } \\
\text { of the user such that they experience feeling immersed in the virtual scene. The environment can } \\
\text { be multisensory in that it can further provide auditory stimuli and haptic feedback, with the latter } \\
\text { being less common. (see Gigante, } 1993 \text { and video example) }\end{array}$ \\
\hline Wearables & $\begin{array}{l}\text { Devices that can be worn on the body (e.g. wristband or chest strap) with the capacity to monitor the } \\
\text { participant's symptoms or anxiety levels (e.g. using physiological indicators) remotely (e.g. outside } \\
\text { of the laboratory/hospital), such that they are wireless and record real-time data. Examples include } \\
\text { the Zephyr BioHarness } 3 \text { and the Empatica E4 }\end{array}$ \\
\hline Electronic screen media (ESM) & $\begin{array}{l}\text { Media for television and computer screens, including computer applications and virtual reality } \\
\text { (Mineo et al., 2009; Shane \& Albert, 2008) }\end{array}$ \\
\hline Telemedicine & $\begin{array}{l}\text { Using information communication technology (ICT) to provide clinical support, overcome geo- } \\
\text { graphical barriers, and to improve health outcomes (World Health Organisation, 2009) }\end{array}$ \\
\hline Ecological momentary assessment (EMA) & $\begin{array}{l}\text { EMA involves participants reporting their subjective experiences and activities during everyday life } \\
\text { using a mobile platform-it is also known as experience sampling methodology (Walz et al., 2014) }\end{array}$ \\
\hline
\end{tabular}

on self-report measures that can lack validity in ASD due to the reasons aforementioned (Macneil et al., 2009). For example, VR can simulate an immersive visual environment with a range of anxiety-evoking stimuli which can induce psychophysiological and behavioural symptoms that can be measured in real-time (Diemer et al., 2014). Similarly, other technology-based approaches can be used to capture realtime anxiety responses: wearables to measure blood volume pulse and galvanic skin response, and ecological momentary assessment (EMA) to measure subjective experiences during everyday life (see Table 1; Hektner et al., 2007). Capturing real-time responses to a range of potentially anxiety-evoking stimuli may help to capture the heterogeneity in ASD, to reduce the possible influence of pre-conceived notions of anxiety, and to detect atypical anxiety triggers and manifestations (Hare et al., 2015).

The most common use of digital technology for the treatment of anxiety is VR exposure therapy as part of cognitive behavioural therapy (CBT), which may have ASD-specific advantages. This is because, rather than asking the patient to think of situations that cause them anxiety, the patient can experience typical and atypical anxiety triggers in VR. VR may facilitate the building of exposure hierarchies by reducing the reliance on the patient's recollection and narration of past experiences, generativity, and imagination-abilities typically impaired in ASD (e.g. Bordignon et al., 2015; Lai et al., 2017; Marini et al., 2018). Further, VR may facilitate the formulation of exposure hierarchies by providing a controlled, repeatable, and diversifiable environment for patients to practice strategies to manage anxiety during a live anxiety-evoking situation in front of the therapist (Newbutt et al., 2016; Parsons et al., 2004). On a practical level, VR can provide a safe setting for exposure therapy, such that any socially inappropriate behaviour exhibited as an atypical anxiety response would not have the same consequences as real-life exposure.

Considering both assessment and treatment, VR and computer-based approaches alike are inherently visual, engaging, and structured and thus in-fitting with ASD-specific recommendations for adapting CBT (NICE, 2013) and for aiding communication during anxiety assessment for autistic individuals (Attwood, 2006). Furthermore, autistic individuals tend to show an affinity towards electronic screen media (ESM), which includes computer applications and VR (Mineo et al., 2009; Shane \& Albert, 2008). Additionally, computer-based and telemedicine approaches allow assessments/treatments to be delivered at-home which may be preferred by autistic individuals (Hepburn et al., 2016) due to not requiring being in an unfamiliar environment, travelling nor transitioning between contexts, and potentially involving fewer interactions with new people, all of which can be challenging or anxiety-evoking for autistic individuals (e.g. Evans et al., 2005; Goodall, 2018; Rezae et al., 2019; Rydzewska, 2016). Because of this, and since technology can facilitate the delivery of self-help tools, technology-based approaches can improve access to mental health care (Hollis et al., 2018), which has been inadequate in the autistic community (Lake et al., 2014; Vogan et al., 2017). Despite the potential benefits of technology-based assessment and treatment approaches for targeting anxiety in ASD, no reviews to date have examined this. Yet, numerous reviews have shown technology-based approaches hold 
promise for targeting anxiety generally (e.g. Cieślik et al., 2020; Grist et al., 2019; Walz et al., 2014) and for targeting functioning in ASD such as social communication skills (e.g. Knutsen et al., 2016; Koumpouros \& Kafazis, 2019; Sandgreen et al., 2020; Valentine et al., 2020).

\section{Aims and Objectives}

The present review therefore aims to fill this research gap by narratively synthesising research on technology-aided assessments and treatments of anxiety in autistic individuals. This consists of four objectives: (1) identify the digital technology used to aid assessments and treatments used for anxiety in autistic individuals, as well as the assessments and treatments themselves; (2) identify the types of anxiety disorders and symptoms targeted in these studies; (3) synthesise and critique the quality of evidence regarding effectiveness, feasibility, acceptability, safety, and utility of technologyaided assessments and treatments in these studies; and (4) identify possible research avenues for future studies in this area. An ancillary objective will be to determine how well characterised samples are with respect to socio-demographic variables, IQ, anxiety diagnosis, anxiety severity, adaptive functioning, social communication skills, and mental health comorbidities (to inform objectives 2, 3, and 4, including gauging the generalisability of synthesised findings).

\section{Methods}

\section{Study Design}

A systematic review was conducted. Reporting guidelines from the Preferred Reporting Items for Systematic Reviews and Meta-Analyses (PRISMA; Moher et al., 2009) and the Synthesis Without Meta-analysis (SWiM; Campbell et al., 2020) were followed. The PRISMA and SWiM checklists are included in Online Resources 1 and 2. For a justification of the chosen methodology, see Online Resource 3.

\section{Protocol and Registration}

Details of the protocol for this systematic review that was registered prospectively on PROSPERO can be accessed at https://www.crd.york.ac.uk/prospero/display_record.php? $\mathrm{ID}=\mathrm{CRD} 42019142910$. Before registering the protocol, existing PROSPERO records and the Cochrane Database of Systematic Reviews were searched to check existing coverage on the topic. Changes to the protocol are listed and justified in Online Resource 4.

\section{Eligibility Criteria}

This review only included original articles written in English and published/in-press within peer-reviewed journals. Studies were only included if they used empirical methods and included at least 5 human participants with ASD diagnoses. It was further specified that only studies aiming to assess or treat anxiety using digital technology (see Table 1) in autistic participants would be included. While studies were excluded if they solely used computerised/telephone versions of measures due to being commonplace in research, studies for inclusion were required to measure anxiety at least once using standardised quantitative measures, digital task/assessments, or physiological measurements. Lastly, papers on genomics, digital phenotyping, and neurotechnology were excluded.

\section{Information Sources}

Searches were conducted in five electronic databases: Embase, MEDLINE, PsycInfo, Cochrane Library, and Web of Science. Databases were first searched on 16 August 2019 and updated on 27 November 2020.

\section{Search Strategy}

For each key concept identified from the research question (i.e. autism, digital technology, and anxiety), relevant search terms were identified (e.g. autis*, digital* and anxi*) and supplemented after examining keyword lists in seminal papers under each key concept and each concept combination therein, as well as controlled vocabulary across the databases. For each database, any terms that were descriptors (i.e. subject headings) were searched for in the [mesh], title, and abstract fields. The remaining terms were searched for in all text fields excluding the affiliation field and author field. However, since the terms "digital*" and "web*" yielded too many false results, they were searched for in the title and abstract fields only. Only subject headings with subheadings relevant to the research question were exploded. As controlled vocabulary varies database-to-database, search strategies were database-specific. Since Web of Science does not have controlled vocabulary, and due to fewer search fields being available, all terms were searched for in the title, abstract, and author keywords fields (see Table 2). No search filters or limiting commands were used on any databases to promote sensitivity. For the full electronic search strategy, see Online Resource 5.

\section{Study Selection}

During study selection, records were categorised as "eligible", "query", and "not eligible" according to the eligibility 
Table 2 Search strategy for Web of Science

\begin{tabular}{llc}
\hline Concept(s) & Search terms & Results \\
\hline Autism & 1. TS = (autis* OR asperger*) & 96,854 \\
Technology & 2. TS = (digital* OR technolog* OR virtual* OR “augmented realit*” & $21,396,513$ \\
& OR "mixed realit*" OR avata* OR robot* OR computer* OR tele* OR & \\
& internet* OR online* OR on-line OR web* OR ehealth OR e-health & \\
& OR itherap* OR i-therap* OR etherap* OR e-therap* OR mhealth OR & \\
& m-health OR emental OR e-mental OR cybercounsel* OR cyber-coun- & \\
& sel* OR electr* OR mobile* OR smartphone* OR "cell-phone*" OR \\
& cellphone* OR "cellular phone*" OR wearable* OR tracker* OR sensor & \\
& OR sensors OR wireless OR portable* OR wristband* OR wrist-band* & \\
& OR smartwatch* OR smart-watch* OR watch OR monitor OR device*) & \\
Anxiety & 3. TS = (anxi* OR phobi*) & 446,852 \\
Combined & 4. \#3 AND \#2 AND \#1 & 1,255 \\
\hline
\end{tabular}

TS Topic, which encompasses the fields Title, Abstract, Keywords, and "Keywords plus" (a Web of Science algorithm that supplies terms expanded from the record's cited references/bibliography). The terms sensor and watch were not truncated as this yielded too many false results criteria. Following de-duplication, records were screened at title and abstract level. A randomly selected $10 \%$ of records were also blindly screened at title and abstract by an independent researcher. Inter-rater reliability for this initial screening was strong (Kappa $=0.80$; percentage agreement $=99.49 \%$ ). Following this, records categorised as eligible or query were assessed for eligibility using full-text review. Full-text screening was completed independently by the first and second authors with moderate inter-rater reliability $(\mathrm{Kappa}=0.64$; percentage agreement $=82.14 \%$ ). Reliability checks were conducted before the updated search. All discrepancies were resolved through discussion with the third author.

\section{Data Collection Process}

A standardised data extraction form (see Online Resource 6) was developed following guidance from the Centre for Reviews and Dissemination (2008). The form was piloted on 3 studies and supplemented to capture variation in methods. The first author independently completed the data extraction using the form and the second author checked the accuracy of the completed forms, except for the study identified in the updated search which was checked by an independent researcher. Key characteristics of the studies were then tabulated.

\section{Data Items}

\section{Sample}

Data on age, gender, diagnoses including ASD, and country were extracted. Other pre-defined sample characteristics, including those that may be potential confounds such as socio-demographic variables, IQ, anxiety diagnosis, anxiety severity, adaptive functioning, social communication skills, and mental health comorbidities were also extracted to inform the quality assessment and satisfy the ancillary objective of determining how well characterised samples are. Further to this, eligibility criteria, any sample size justifications, sampling methods, and recruitment procedures were extracted to inform the quality assessment.

\section{Phenomenon of Interest}

The type of digital technology used, assessment characteristics (e.g. tasks and assessment tools used), and intervention characteristics (e.g. anxiety targeted, treatment delivered and duration) were extracted.

\section{Design}

The research design employed, and any conditions, was extracted.

\section{Outcomes of Interest}

Anxiety measures were outcomes of interest in terms of treatment effects and whether multi-modal methods were used. Because feasibility and acceptability of the assessments and treatments were of interest, any information on rates of identification, enrolment, recruitment, retention/ drop-out, response/data completion, and on usability (e.g. technical problems) was extracted. For treatment studies, session attendance, treatment completion rates, and therapist fidelity was also extracted.

\section{Research Types}

Since both qualitative and quantitative records could be included, the research type was determined. 


\section{Risk of Bias in Individual Studies}

All authors reviewed the quality assessment tool and agreed adaptations (see Online Resource 7). During quality assessment, each study was assessed using an adapted version of the Effective Public Health Practice Project (EPHPP) tool for quantitative studies. This tool requires rating the risk of sampling bias, selection bias, experimenter/researcher bias, participant bias, attrition bias, or confounding, as well as the quality of study design, data collection tools, the intervention/assessment delivered, and any analysis conducted. Sections covering these aspects (see Fig. 2) are rated as "strong", "moderate", or "weak" in quality per study. Based on these ratings, following the adapted EPHPP instructions, a global rating was computed that was then judged in terms of applicability. The global rating was deemed applicable in all cases. Author 1 and an independent researcher conducted the quality assessments independently on all included papers. Disagreements due to clear oversight were identified and corrected through discussion. At this point, weighted kappa $\left(\kappa_{\mathrm{w}}\right)$ with quadratic weights (Fleiss \& Cohen, 1973) was calculated to determine inter-rater reliability. It showed statistically significant agreement between the two raters for global and section ratings, $\kappa_{\mathrm{w}}=0.762,95 \%$ CI [0.634, 0.891], $\mathrm{p}<0.01$. Percentage agreement was $82.9 \%$. The strength of the agreement was classified as good according to Altman's (1991) classifications and excellent according to Fleiss et al. (2003) classifications. The additional paper yielded in the updated search was independently assessed by author 1 and another party with corrections made. For all papers, disagreements due to differing interpretation were resolved by consensus after consulting authors 2 and 3. One paper was coauthored by author 3 , and so disagreement on this paper was discussed with author 2 only.

\section{Synthesis of Results}

With reference to the quality appraisals, papers' methodologies and results were critically reviewed and combined textually by a formal narrative synthesis. This was informed by guidance from the Economic and Social Research Council (ESRC; Popay et al., 2006). Studies deemed directly relevant to objective 3 were prioritised for drawing conclusions from the synthesis. To address the present review's aim, studies were grouped according to whether they focused on the assessment or treatment of anxiety under each research objective and were further grouped by the main methodology used.

\section{Results}

\section{Study Selection}

An overview of the study selection process is shown in Fig. 1. The database search yielded 3529 records. Following the removal of duplicates $(n=1,101)$, the titles and abstracts of records were screened according to the eligibility criteria, leading to the removal of 2,391 records. After a backward and forward reference search of eligible records, 2 additional records were screened at full-text and accepted. No additional records were identified after searching the reference lists of prior related reviews. The remaining records $(n=36)$ were assessed for eligibility using full-text review and 15 papers that consisted of 16 studies were deemed eligible for inclusion in the narrative synthesis.

\section{Synthesis of Study Characteristics and Results}

For a summary of the studies' key characteristics and results, see Tables 3, 4, 5, and 6 .

\section{Sample Characteristics}

Apart from one study that recruited adults only (i.e. Hare et al., 2015), all assessment studies recruited adolescents with one recruiting adults as well (i.e. Chen et al., 2016). The overall age range for assessment studies was 10 to 61 years. Treatment studies recruited children and adolescents (age range: 3 to 19 years), except for two that recruited adults only (i.e. Gaigg et al., 2020; Maskey, et al., 2019c; age range: 18 to 66 years). Approximately $81 \%$ of participants across all samples were male, though some studies only reported demographics of their final sample and Suresh and George (2019) did not report the proportion of males. None of the assessment studies required participants to show clinically significant anxiety levels to participate, but for most samples a substantial proportion did and for one study this was unreported (i.e. Liu et al., 2008). Samples in most of the treatment studies had clinically significant anxiety (i.e. Conaughton et al., 2017; Hare et al., 2016; Hepburn et al., 2016; Maskey et al., 2014, 2019a, b), even for the three studies for which this was not an inclusion criterion (i.e. Gaigg et al., 2020; Hare et al., 2016; Maskey et al., 2014). In the remaining treatment studies, this was not reported. Most studies did not report comorbidities or excluded on this basis (e.g. severe mental health disorders), and those that did typically reported multiple anxiety diagnoses and low mood. Studies were mostly based in the UK, except for 1 in India (i.e. Suresh \& George, 2019), 4 in the USA (i.e. Hepburn et al., 2016; Isong et al., 2014; Johnson et al., 


\section{श्राISMA PRISMA 2009 Flow Diagram}

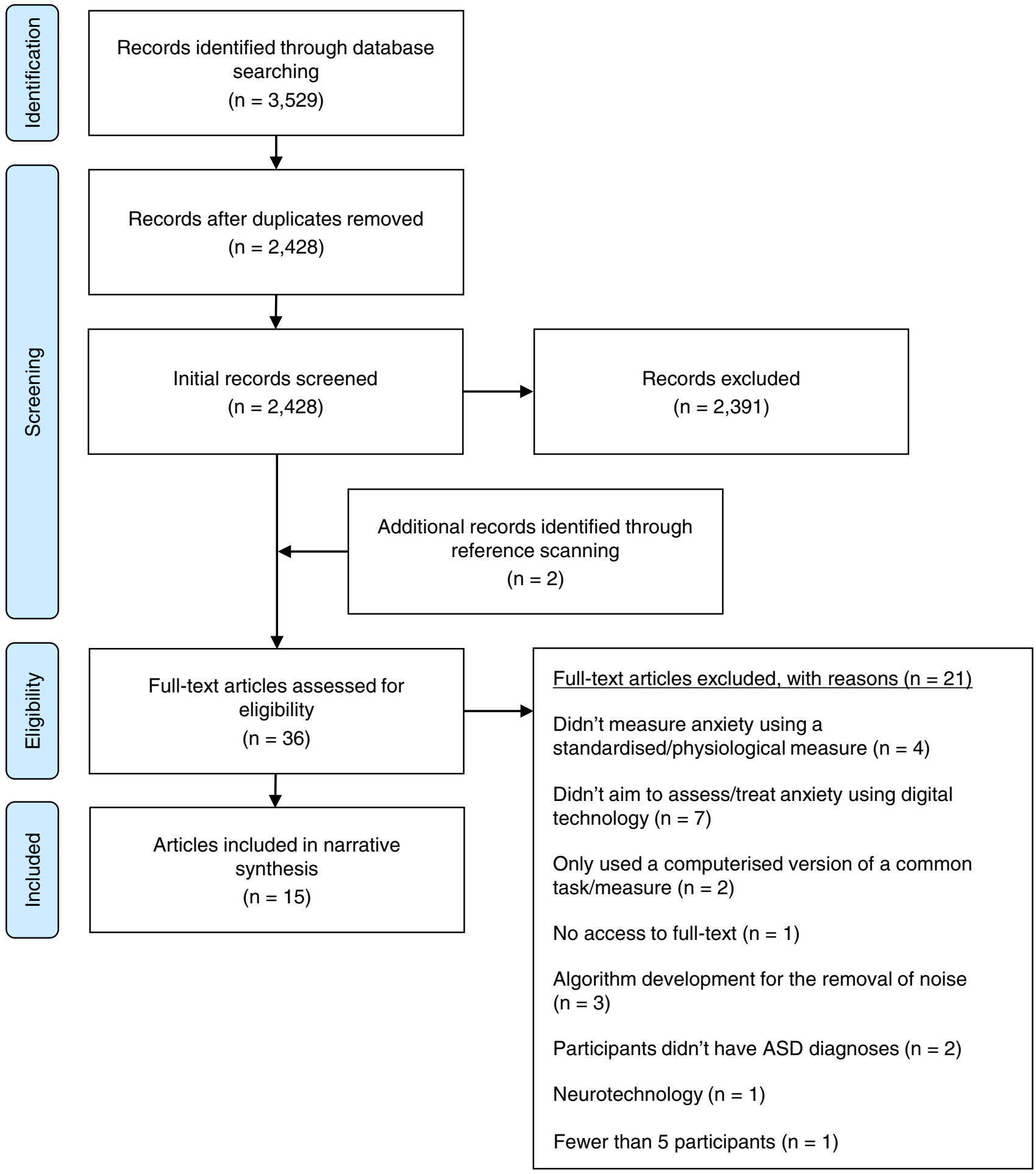

Fig. 1 PRISMA 2009 Flow diagram 


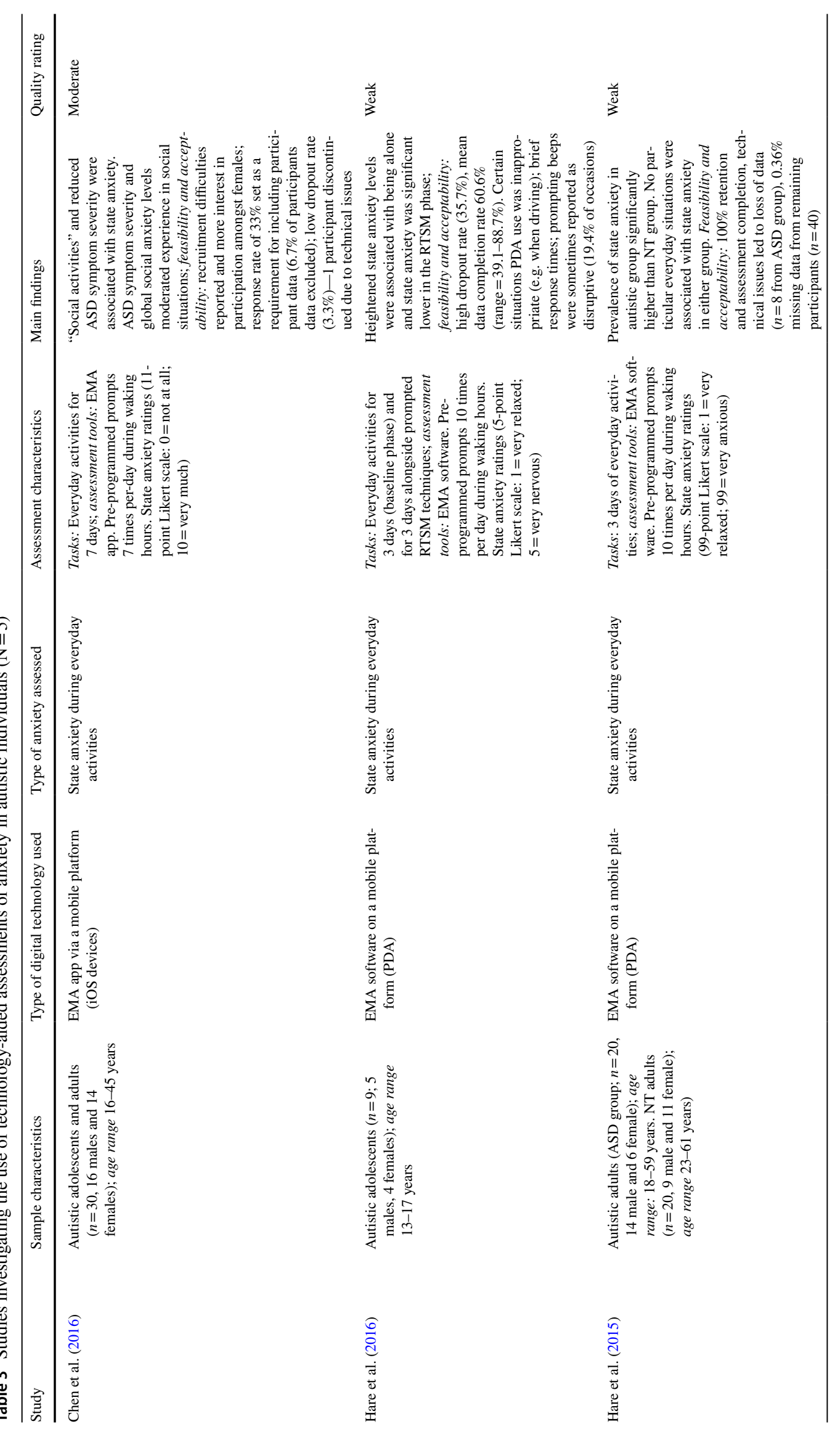




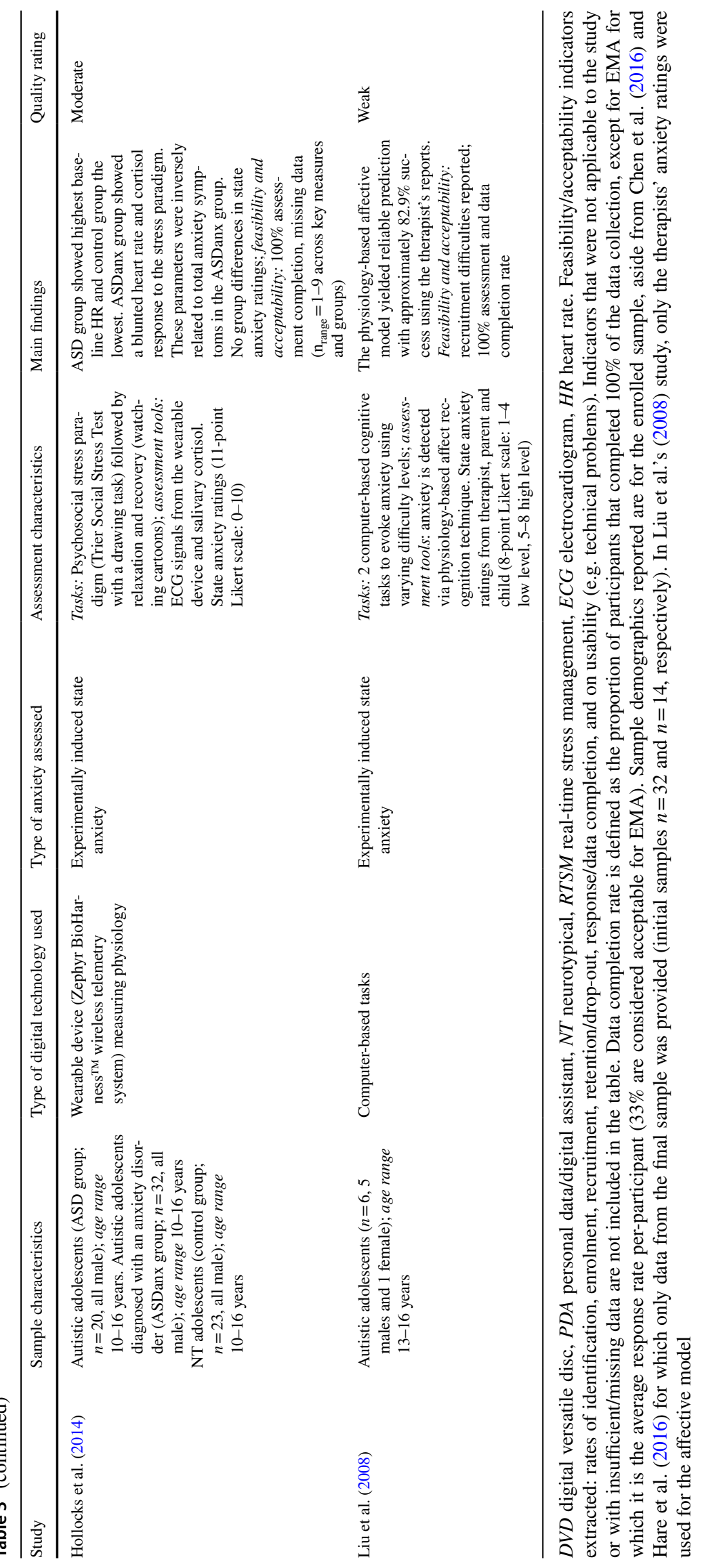




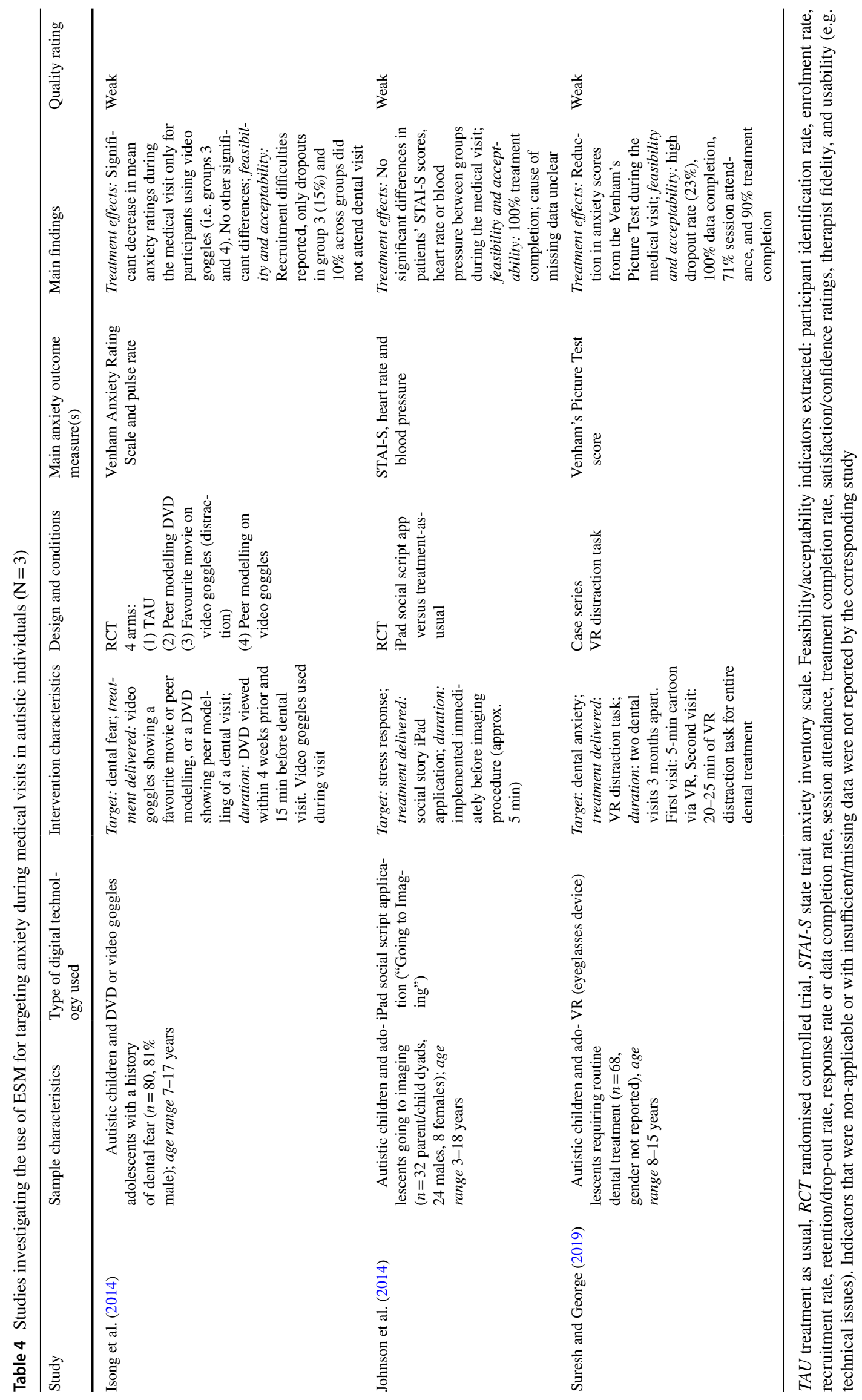




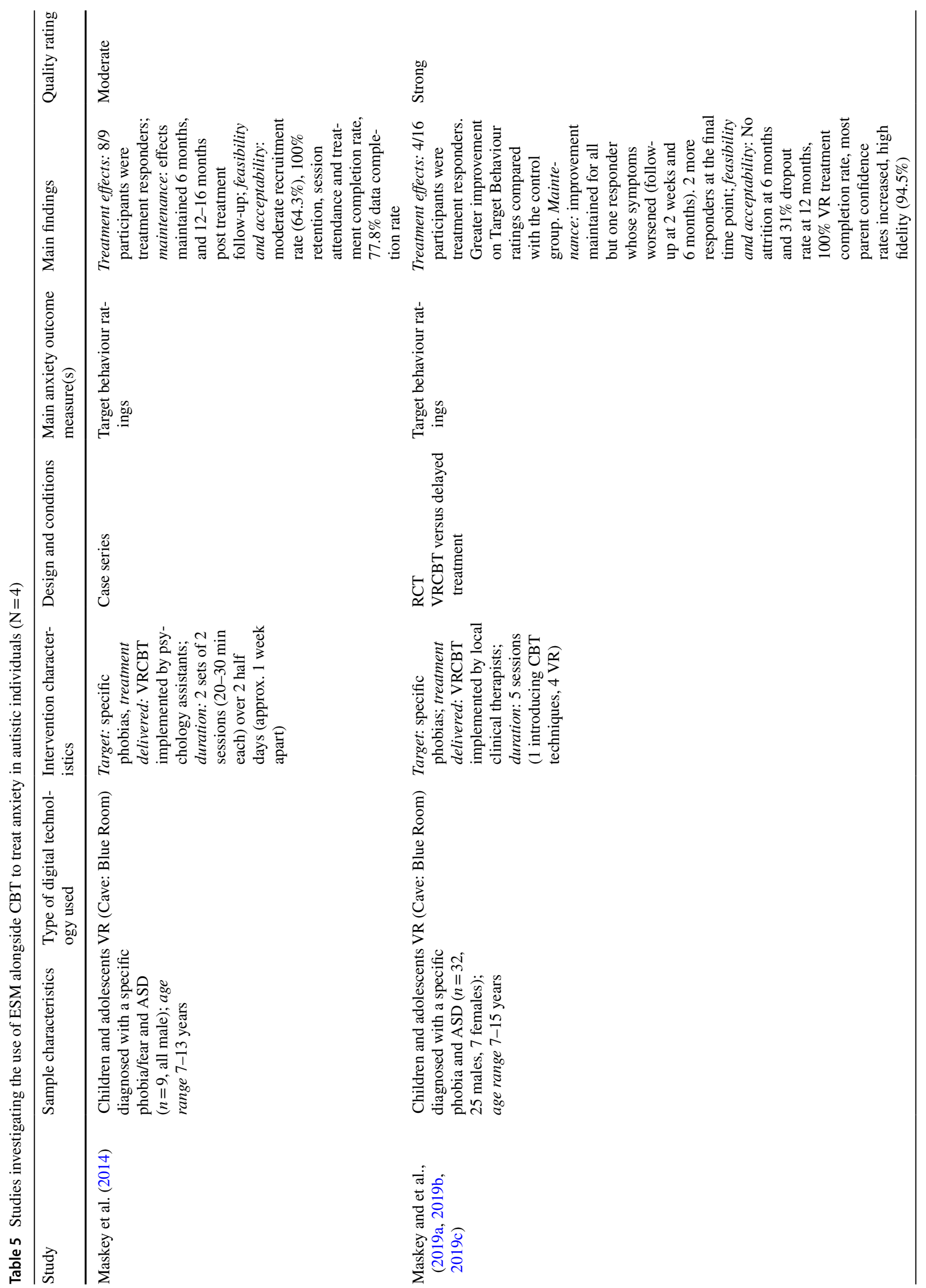




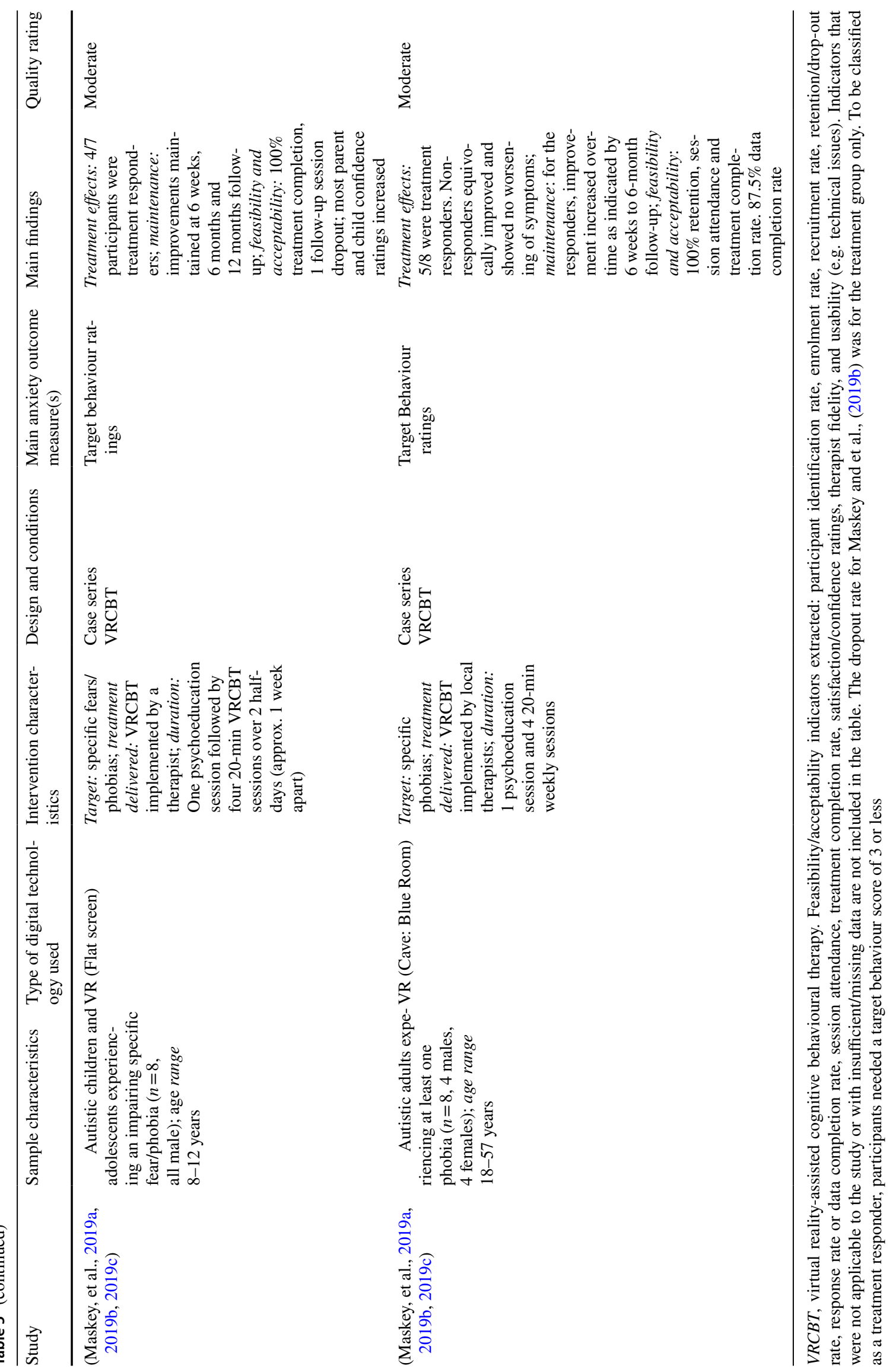




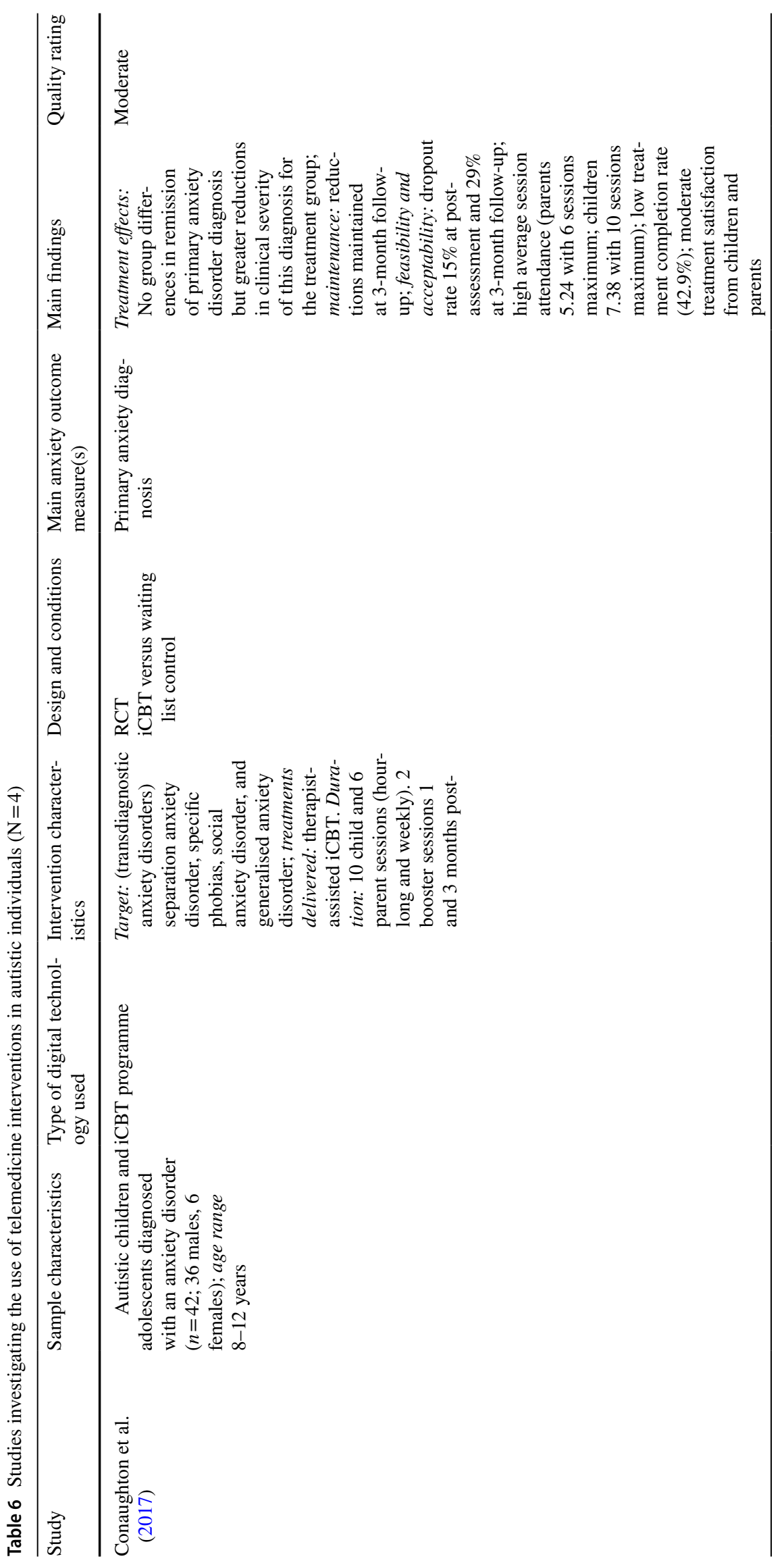




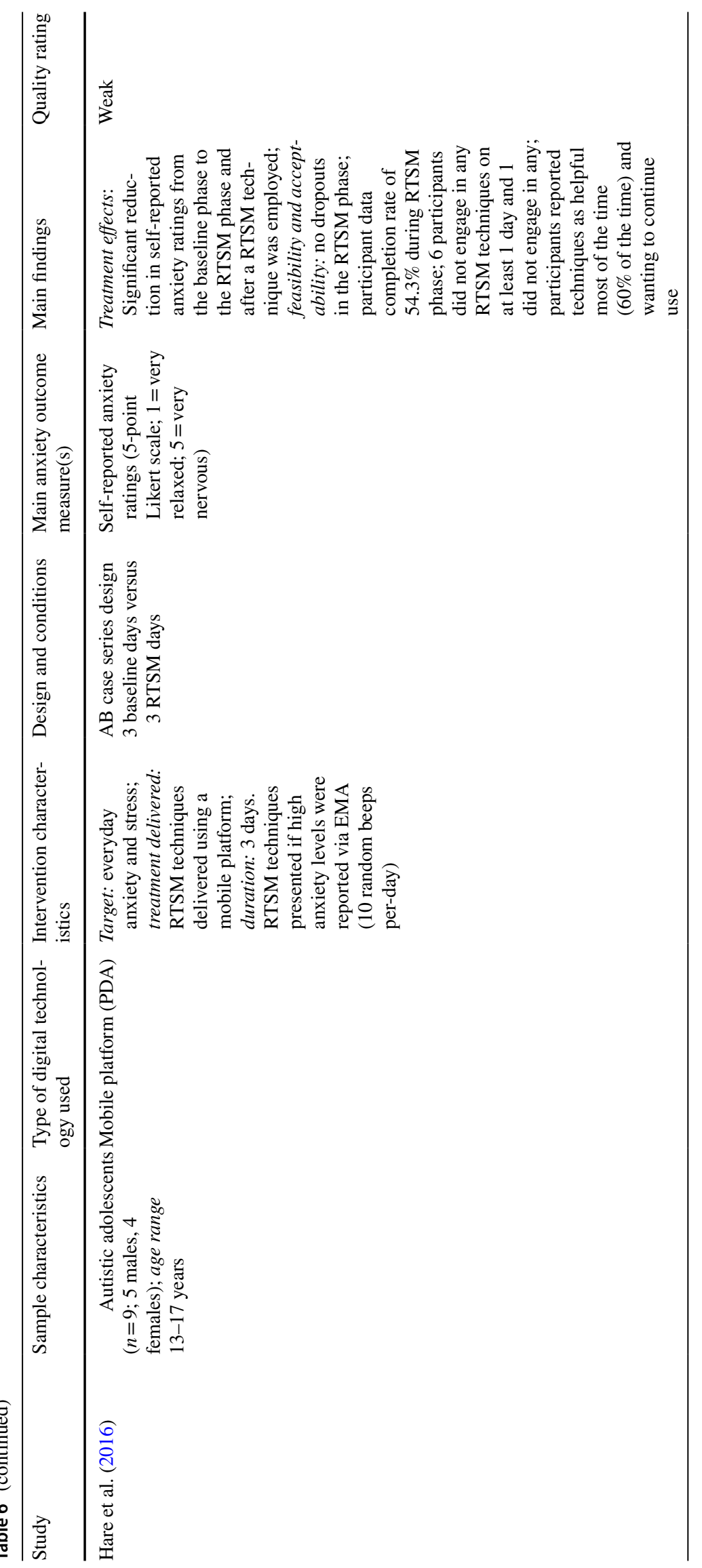




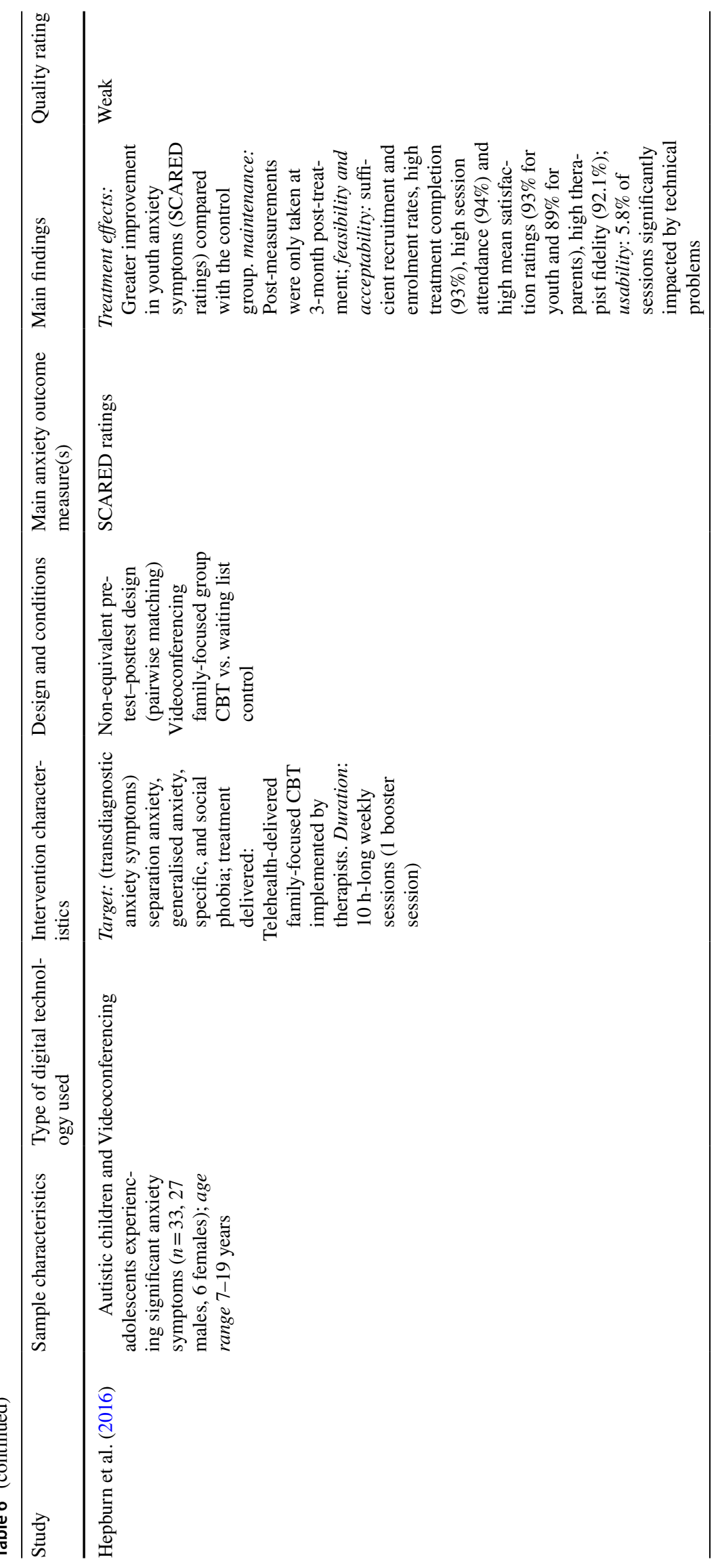




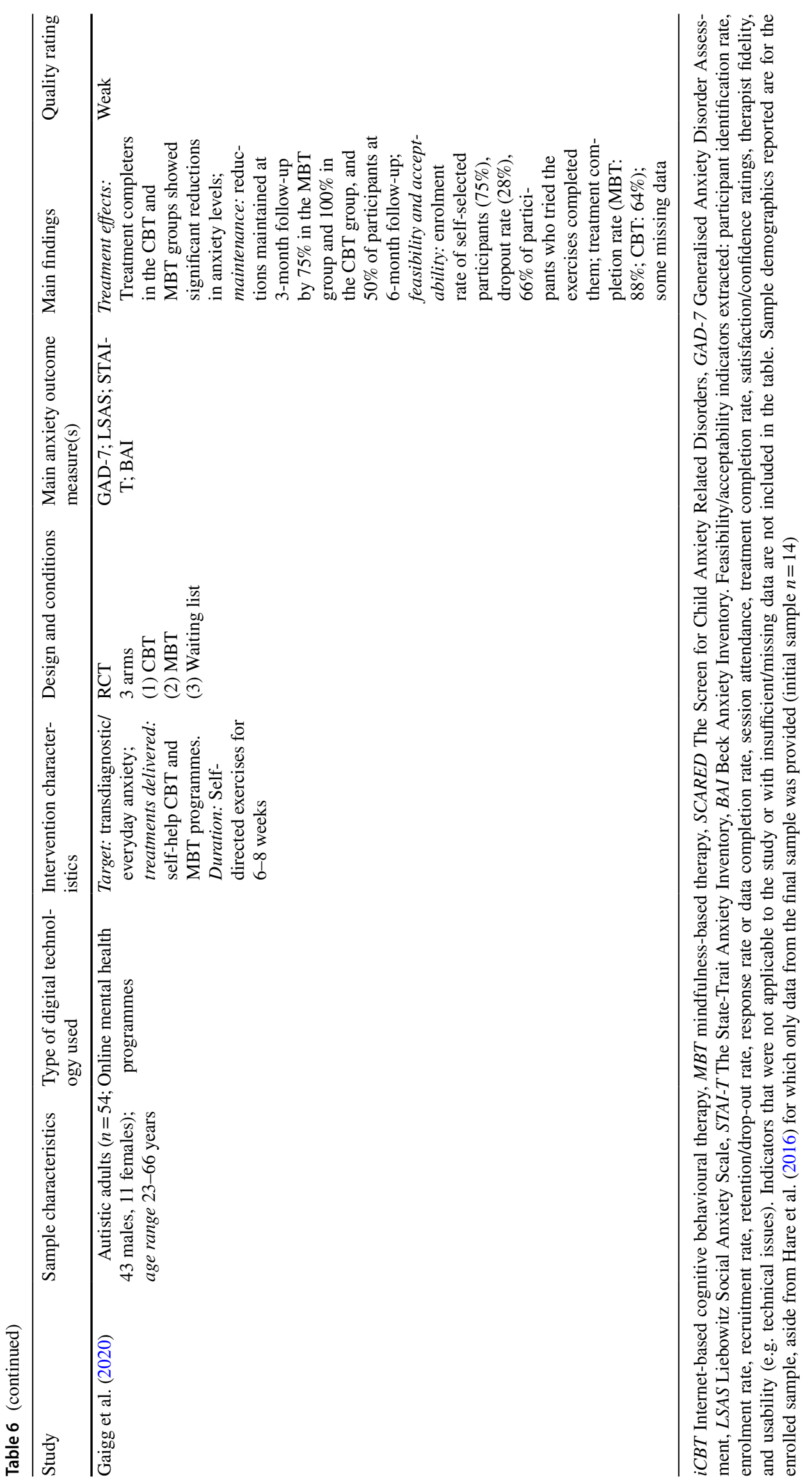


Fig. 2 Quality assessment ratings for assessment studies per category from the Adapted EPHPP Tool. Note. Stronger quality ratings indicate reduced risk of bias

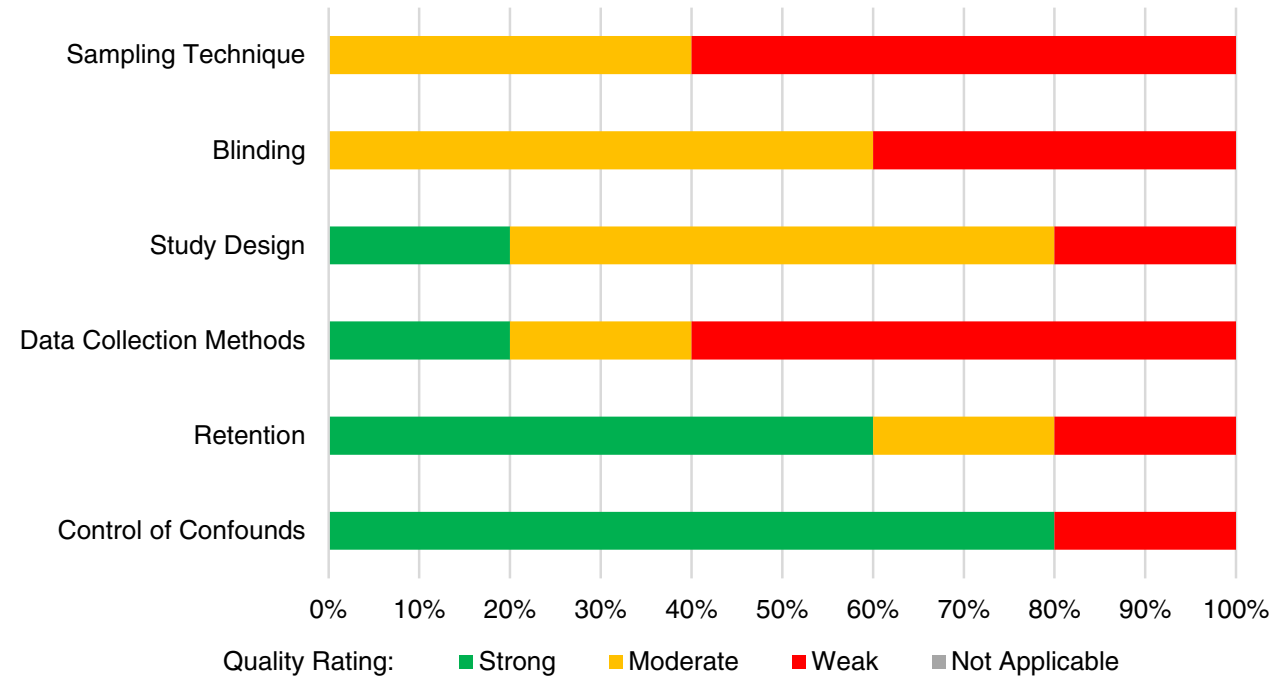

2014; Liu et al., 2008), 2 in Australia (i.e. Chen et al., 2016; Conaughton et al., 2017), and some of Chen et al.'s (2016) participants were based in Taiwan.

\section{Risk of Bias within Studies}

As indicated in Figs. 2 and 3, no studies were rated strong with respect to sampling technique or blinding. Around half of all studies were moderate in quality with respect to sampling technique as their samples were considered at least somewhat likely to be representative, but since the rest of studies showed a high risk of selection bias, they were rated as weak in this respect. With respect to blinding, two factors were considered: (1) the risk of reporting bias and (2) the risk of researcher bias for assessment studies and detection bias for treatment studies. The risk of reporting bias was high in all studies as it could not be determined if participants were aware of the research question in any.
This was coupled with a high risk of researcher or detection bias for most studies, hence why they were rated as weak in terms of blinding. Assessment studies were considered to exhibit high risk of researcher bias if they did not report whether researchers/evaluators present during assessment task were blind to the aims of the study. Treatment studies were considered to be at high risk of detection bias if outcome assessors were not blinded or this was not reported. The remaining studies were moderate with respect to blinding as detection/researcher bias was deemed unlikely.

Most designs were strong or moderate due to being deemed suitable for testing the research questions/hypotheses. However, because most treatment studies aimed to test feasibility and only 5 studies conducted randomised controlled trials (RCTs; potentially underpowered), they only provide a preliminary indication of efficacy. Additionally, the data collection methods belonging to around half of all studies were weak either because they were not valid, or both
Fig. 3 Quality assessment ratings for treatment studies per category from the Adapted EPHPP Tool. Note. Stronger quality ratings indicate reduced risk of bias

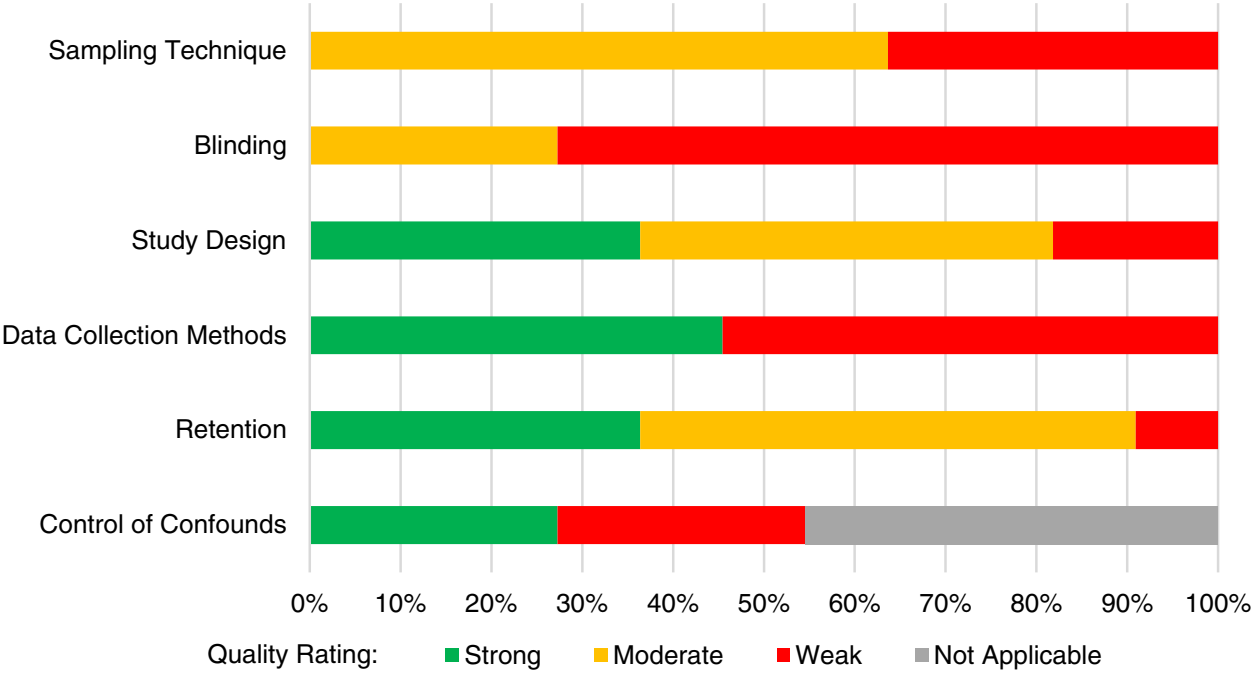


their validity and reliability could not be determined. While around half of studies showed high study completion rates of $80 \%$ or more and so were rated strong in quality for lack of retention, the remaining half showed lower completion rates, or the number of withdrawals and drop-outs was unreported. For most studies with potential confounding variables, these were often controlled or accounted for and so strong in this respect.

Ratings of the categories in Figs. 2 and 3, alongside consideration of the quality of intervention/assessment integrity and analyses using the adapted EPHPP tool, were used to inform the global rating for each study. As shown in Tables 3, 4, 5, and 6, most $(\mathrm{N}=12)$ studies were rated weak, some $(\mathrm{N}=3)$ studies were rated moderate, and 1 was rated strong.

\section{Assessment Studies: Synthesis of Results and Strength of Evidence}

Technology-based assessments of anxiety in ASD show promise for assessing state anxiety in everyday life and labbased settings, but recurring issues amongst the assessment studies include the use of self-report scales that lack validity in ASD alongside lack of controlling for inter-individual differences that could act as potential confounds. Further, the generalisability of findings to the broad autistic population is questionable due to involving small convenience samples $(n \leq 30)$ of mainly adolescents, with at least average verbal intelligence, absence of reporting on participant enrolment and recruitment rates, and the variability in retention rates. Overall, these limitations notwithstanding, results collectively indicate that anxiety in ASD may have a distinct psychophysiological signature and be evoked by idiosyncratic triggers.

\section{Everyday State Anxiety}

Three of the assessment studies used EMA delivered via a mobile platform to assess everyday anxiety in autistic individuals. Participants received daily pre-programmed prompts to self-report their level of state anxiety, via a rating on a Likert scale (see Table 3), as well as the activity they were engaging in. Chen et al. (2016) used purely quantitative methods, whereas the others used mixed methods that included a qualitative exploration of the phenomenology of reported thoughts/anxiety. Studies produced conflicting findings regarding the type of everyday activities associated with heightened state anxiety. Chen et al. (2016) found elevated state anxiety to be associated with social activities, Hare et al. (2016) with being alone, and Hare et al. (2015) with no particular activity. As can be seen in Table 3, the cause of these conflicting findings may be attributable to differing methodologies since the way activities were coded, the range of the Likert scales used for self-reporting state anxiety, the age of participants, and the number of sampling days, varied between the studies. Crucially, while Hare et al., (2015, 2016) reported that autistic participants showed clinically significant levels of anxiety, neither study reported participants' levels of social anxiety which Chen et al. (2016) found to have a moderating effect. Chen et al.'s (2016) findings are perhaps the most certain as the study was moderate in quality. Chen et al.'s (2016) findings must however be interpreted with caution considering the use of multilevel modelling with a small sample size $(n=30)$ and that, in contrast to the other EMA studies, inter-rater reliability for the coding of activities and thoughts was not calculated.

Findings did however consistently suggest that everyday state anxiety appears to be distinct in ASD in terms of its variability and idiosyncratic phenomenology (Hare et al., 2016), its increased presence (Chen et al., 2016; Hare et al., 2015), and its associated cognition (Hare et al., 2015). However, only Hare et al. (2015) demonstrated the distinctness of anxiety in ASD via group comparisons between autistic and neurotypical individuals (i.e. using a mixed design), with the other studies solely recruiting autistic participants (i.e. using a within-subjects design). Moreover, the group differences found by Hare et al. (2015) could instead be attributed to trait anxiety, rather than ASD, as the autistic group showed significantly higher anxiety levels. In terms of the validity of assessing anxiety using EMA in ASD, this remains unclear predominately because these studies relied solely on selfreport ratings of state anxiety that can lack validity in ASD.

These studies indicate that EMA may be feasible and acceptable for assessing everyday anxiety in ASD, but recruitment and technical issues need addressing. While most participants in Hare et al.'s (2015) and Hare et al.'s (2016) studies found the technology acceptable, this was not reported on in Chen et al.'s (2016) study though the response and retention rates were high with only one participant dropping out due to technical issues. Lastly, Hare et al.'s (2016) participants suggested that participant-initiated EMA, as opposed to the pre-programmed prompts, may be more acceptable due to being less disruptive. These suggestions may however have been impacted by another component of Hare et al.'s (2016) study that increased participant burden. That is, in the second stage of the study, participants were asked to employ real-time stress management (RTSM) techniques if they rated their state anxiety as high (see Treatment section for more details).

\section{Experimentally Induced State Anxiety}

The two remaining assessment studies used quantitative methods, experimental designs, and measured physiological responses in autistic individuals during laboratory-based tasks designed to induce stress. The digital technology used 
in these two studies included a wearable device to measure physiology (Hollocks et al., 2014) and computer-based cognitive tasks (delivered via a computer monitor) for stress induction (Liu et al., 2008). Physiological parameters included heart rate and heart-rate variability. Both studies found physiological responses to be related to anxiety levels but in different respects due to the methodology employed. Liu et al. (2008) generated a physiology-based affective model that reliably predicted therapist-reported state anxiety, whereas Hollocks et al. (2014) did not report therapists' ratings of state anxiety and showed a more nuanced pattern of findings. Compared to Liu et al. (2008) who did not report whether participants showed clinically significant anxiety, Hollocks et al. (2014) conducted group comparisons according to whether participants met criteria for an anxiety disorder and/or for ASD. For the group with anxiety and ASD diagnoses, it was uniquely found that physiological responses were inversely associated with anxiety symptoms, but these differences were not mirrored by parental- or childreported levels of state anxiety during the task. Liu et al.'s (2008) results should be interpreted with caution as only therapists' reports of state anxiety were used in the analysis, and neither blinding, participants' levels of trait anxiety, nor presence of any anxiety diagnoses were reported. Most fundamentally, the suitability of the methodology and the pattern of results were difficult to discern due to unclear reporting hence why it was rated as weak. Since only Hollocks et al.'s (2014) findings provide sufficient certainty, it can only be concluded that autistic individuals with anxiety diagnoses likely show a blunted physiological stress response, but the shared limitations of the assessments studies did apply here.

Because neither study aimed to gauge feasibility and acceptability, which is not unusual for an assessment study, only some of these indicators were reported. All participants in both studies completed the assessment, although missing data in Hollocks et al.'s (2014) study and recruitment difficulties in Liu et al.'s (2008) study were reported. For now, it is challenging to generalise findings to the broader ASD population due to the limitations of both studies, but they do indicate that physiological parameters for anxiety assessments may be useful in potentially bypassing the aforementioned problematic validity of self-report scales in ASD, and Liu et al.'s (2008) study provides some indication that computerised tasks may be suitable for inducing state anxiety in ASD.

\section{Treatment Studies: Synthesis of Results and Strength of Evidence}

Two main types of digital technology were used for targeting anxiety in ASD: ESM and telemedicine (see Tables 4, 5, and 6). Contrary to the assessment studies, most treatment studies provided key feasibility indicators, and all were quantitative. As per Tables 4, 5, and 6, there were three research designs employed in the treatment studies: RCTs $(\mathrm{N}=5)$, non-equivalent pre-test post-test design $(\mathrm{N}=1)$, and case series $(\mathrm{N}=5)$. While it is difficult to summate results due to the clinical and methodological heterogeneity, especially with respect to the nature of the interventions, these studies provide preliminary evidence for technology-based interventions being efficacious in reducing anxiety in ASD and for some maintenance of treatment effects. Only interventions involving peer modelling and social scripts did not show promise. Furthermore, the representativeness of the samples is likely constrained by the use of convenience samples of young people typically with verbal IQs of $\geq 70$, and because most studies employed either potentially underpowered- or non-RCTs and/or focused on feasibility, treatment results can only provide a preliminary indication of the efficacy. Alongside this, in two of the strongest studies and the only two to test technology- and therapist-assisted CBT in an RCT, improvements did not translate into loss of the targeted anxiety diagnosis for around $62 \%$ of participants. Overall, aside from technical issues and some recruitment and retention issues reported, interventions were shown to be feasible and acceptable.

\section{Electronic Screen Media}

ESM was used to target anxiety by displaying a demonstration of a situation likely to be anxiety-evoking or as a relaxation/distraction task. Isong et al. (2014) and Johnson et al. (2014) used visual media (i.e. images and videos) to aid learning of medical visit procedures, via peer modelling and social scripts respectively, and were the only studies to involve interventions found not to reduce anxiety levels. However, for the group of Isong et al.'s (2014) participants that viewed the peer modelling via video goggles, rather than a DVD (i.e. television screen) as used by the other group or an iPad as used by Johnson et al.'s (2014) participants, the intervention was found to reduce anxiety levels. A further group of Isong et al.'s (2014) participants, for whom the assigned intervention reduced anxiety levels, wore video goggles to watch a favourite video as a distraction task during a medical visit. Likewise, Suresh and George (2019) successfully employed a distraction task for the same purpose but simulated in VR. In the remaining studies, VR was used to reduce a specific fear/phobia, as opposed to merely anxiety during medical visits like the others, via relaxation and exposure tasks constituting CBT (i.e. Maskey et al., 2014, 2019a, b, c). The majority of participants across the virtual reality-assisted cognitive behavioural therapy (VRCBT) studies were classed as treatment responders due to showing reduced specific fear/phobic behaviours post-treatment. Typically, treatment effects showed maintenance (see Table 5). 
Commonly, interventions showing promise for reducing anxiety in ASD were those that used ESM for exposure, relaxation, or distraction. However, in the only VRCBT study that was an RCT instead of a case series and that was rated strong rather than moderate (Maskey, et al., 2019b), the majority of participants were not classed as treatment responders, and self- and parental-reported anxiety scores did not differ significantly between groups nor over time. Still, there were statistically significant improvements on the main anxiety outcome (Target Behaviour Ratings) for the treatment group. Nonetheless, all other studies using ESM (i.e. non-VRCBT studies addressing medical visit anxiety) were weak in quality. Isong et al. (2014) and Suresh and George (2019) included additional flaws that collectively diminish the certainty of efficacy findings. That is, psychopharmacological medication usage was unknown, which is a potential confounding variable and the analyses were unclearly reported.

The generalisability of feasibility and acceptability results is constrained by the variation in whether participants met the diagnostic criteria for a specific phobia. Furthermore, most ESM studies did not report recruitment rates, none reported participant satisfaction nor whether technical issues were experienced, and data completion rates were missing or variable. However, VRCBT was shown to be feasible and acceptable in that there was a $100 \%$ treatment completion rate and only 1 dropout at follow-up. This was not so apparent for studies targeting anxiety during medical visits as dropout and treatment completion rates tended to be less favourable and recruitment difficulties were reported by Isong et al. (2014). Additionally, while the VRCBT findings are promising, their generalisability is compromised by the eligibility requirement for participants to be interested in the intervention and to be experiencing a fear/phobia deemed fit for simulating in the VR environment, but due to lack of reporting it is difficult to determine which fears/phobias may be inappropriate and the likely degree of interest within this patient group.

\section{Telemedicine}

All four telemedicine interventions appeared to reduce anxiety levels in autistic individuals with clinically significant anxiety. Interventions involved Internet-based cognitive behavioural therapy (iCBT; Conaughton et al., 2017), CBT delivered via videoconferencing (Hepburn et al., 2016), online self-help CBT and mindfulness-based therapy (MBT) programmes (Gaigg et al., 2020), and RTSM delivered via a mobile platform (Hare et al., 2016). In the latter study by Hare et al. (2016), participants reported how anxious they felt at random intervals in the day when prompted to do so by their mobile device (i.e. using EMA) and if they rated their anxiety levels as high, the platform presented instructions for a range of common RTSM techniques could select from. Results must be interpreted with caution; however, as no telemedicine studies were rated as strong, none included active comparators, and Hare et al. (2016) had no control group. Additionally, only Conaughton et al. (2017) was rated as moderate and used multi-modal and multi-informant measures of anxiety that are more likely to produce valid results in autistic samples. Still, while Conaughton et al. (2017) found no group differences in their main anxiety outcome measure, remission of primary anxiety diagnosis, the iCBT group did show greater reductions in the clinical severity of the primary anxiety diagnosis, the number of anxiety symptoms and disorders, as well as improvements in functioning across diverse indicators. Furthermore, Conaughton et al.'s (2017) and Gaigg et al.'s (2020) follow-up and Hepburn et al.'s (2016) post-treatment measurements at 3 months indicated maintenance of treatment effects. However, only $50 \%$ of participants in Gaigg et al.'s (2020) 6-month followup showed maintenance and the waiting-list control group likewise showed reductions in anxiety over time such that at this timepoint active treatment showed no advantage.

In terms of feasibility and acceptability, only Hepburn et al. (2016) reported their recruitment rate, although it was deemed feasible and all studies reported low dropout rates during the treatment phase and most reported at least moderate treatment satisfaction. Conaughton et al. (2017) and Hepburn et al. (2016) found high session attendance, but a substantial proportion of participants in Gaigg et al.'s (2020) did not complete the self-help programme nor return requested diary records pertaining to the use of strategies, and in Hare et al.'s (2016) study did not engage in any RTSM techniques on at least 1 day. However, it is unclear whether this latter finding was a result of participants not rating their state anxiety as high which would prevent the RTSM techniques from being presented (i.e. triggered) on the device at all. Lastly, technical issues caused dropout in the baseline phase of Hare et al.'s (2016) study and impacted some sessions in Hepburn et al.'s (2016) study, but this was not reported on by Gaigg et al. (2020) or Conaughton et al. (2017) which might otherwise explain the low treatment completion rate in the latter study.

\section{Discussion}

This review aimed to narratively synthesise research on technology-aided assessments and treatments of anxiety in autistic individuals for the first time. Despite the longestablished link between anxiety and ASD, and the potential of digital technology to facilitate ASD-specific adaptations for its assessment and treatment, searches yielded only 16 studies. This area has thus been neglected relative to the preponderance of research outside of ASD that supports the 
use of digital technology for aiding the assessment and treatment of anxiety (see reviews by Cieślik et al., 2020; Gujjar et al., 2019; Walz et al., 2014).

\section{Summary and Critique of Evidence: Assessment Studies}

While there were only five assessment studies, collectively they indicate the nature of anxiety to be distinct in ASD, as demonstrated by non-technology-based research (e.g. Lau et al., 2020; Magiati et al., 2017). Three of the assessment studies used EMA to assess everyday state anxiety (i.e. Chen et al., 2016; Hare et al., 2015, 2016) indicating it to be distinctly characterised, pronounced, variable, and idiosyncratic in ASD. ASD-specific characteristics included rumination, worries regarding the need for rules and coping with change, confusing and self-focused thoughts. These results are congruent with the wider ASD literature (e.g. Bearss et al., 2016; Lau et al., 2020; Ozsivadjian et al., 2012; Robertson et al., 2018). Conversely, review findings are incongruent with this literature base in that anxiety was not commonly shown to be triggered by social situations. This may be explained by the small samples or the fact that only one study (i.e. Chen et al., 2016) controlled for social anxiety levels which had a moderating effect. Equally, these mixed findings may be attributable to the extensive methodological variation. Similarly, the inference drawn from these EMA studies, that anxiety is distinct in ASD, is potentially spurious because only Hare et al. (2015) included an autistic and neurotypical group for comparison, and the autistic group showed significantly higher and clinically significant levels of anxiety that could have confounded results. Moreover, the studies relied solely on self-report measures of anxiety, which are known to lack validity in ASD (Macneil et al., 2009). These potential validity issues notwithstanding, as concluded in the non-ASD literature (see Walz et al., 2014), assessing anxiety using EMA can be insightful but its burdensomeness should be considered. Future studies should follow Santangelo et al. (2013) comprehensive guidance to address such issues. EMA may offer particular value to this field due to it being well-suited to capture inter-individual and situational variation in anxiety triggers and symptoms (Walz et al., 2014), and in everyday thoughts, feelings, and behaviours of this characteristically heterogenous population, at least in individuals with high cognitive functioning (Hare \& Chen, 2019).

As a means of future studies reducing their reliance on subjective measures of anxiety, physiological responses could be captured passively during everyday activities using wearables. This may prove synergistic in enhancing our understanding of anxiety in ASD, as has been apparent beyond ASD (Walz et al., 2014). Only one reviewed study (i.e. Hollocks et al., 2014) used a wearable to measure physiology-based anxiety, and this was during a laboratory-based stress induction task. It was found that autistic participants with anxiety diagnoses showed a blunted stress response relative to controls, indicative of chronic autonomic hyperarousal which has since been demonstrated in autistic individuals experiencing emotional difficulties (Patriquin et al., 2019). However, autonomic responsivity in individuals with anxiety diagnoses has been shown to vary as a function of the test and stimuli used, with ecologically valid assessment tools more likely to evoke hyperactivity (Hu et al., 2016). This ought to be investigated in ASD, especially considering conclusions from a recent review that, due to mixed findings, the physiological markers of anxiety in ASD remain uncertain but their identification may permit a nuanced assessment that can thus accommodate for the heterogeneity in ASD (McVey, 2019). With respect to the acceptability of wearables in autistic participants, while Hollocks et al. (2014) did not aim to investigate this and participants were only required to use the wearable for a limited period in a laboratory, assessment completion rates were high. A more recent review directly investigated this across a range of wearables and found that obtrusive devices including the chest strap device used by Hollocks et al. (2014) were least suitable for autistic individuals, and that less obtrusive wrist-worn devices were preferred by participants and comparable in terms of clinical validity (Taj-Eldin et al., 2018), but it must be considered that less obtrusive devices tend to provide less accurate data.

In summary, although more research is required to reach a definitive conclusion and to overcome the limitations common to all reviewed studies that are discussed later, the assessment studies collectively indicate that autistic individuals' psychophysiological stress response may have a distinct signature that varies according to the presence of anxiety symptoms and can be feasibly assessed in lab-based and everyday settings. A complementary area requiring research attention is the use of digital technology to experimentally induce and thus assess state anxiety under controlled conditions. Only Liu et al. (2008) tested this, using a computerised cognitive task, and limitations in the reporting of the methodology rendered it difficult to draw firm conclusions from this study. Relatedly, no studies were identified that tested the use of VR for assessing anxiety except in the context of VR exposure therapyan area lacking research outside of ASD despite its wellrecognised potential (Firth et al., 2018; Freeman et al., 2017). Generally, while computerised anxiety-induction tasks for use in laboratory-based studies are increasingly being adopted, there is a relative paucity of studies investigating the use of digital technology to assess anxiety (Firth et al., 2018; Walz et al., 2014). 


\section{Summary and Critique of Evidence: Treatment Studies}

Technology-based interventions appeared to broadly reduce anxiety levels in autistic individuals. Findings are analogous with the general literature in terms of the types of technologies and interventions used, the anxiety targeted, and outcomes achieved (e.g. Cieślik et al., 2020; Grist et al., 2019; Gujjar et al., 2019). Findings similarly correspond with the ASD-specific literature in that technology-aided interventions, involving overlapping technologies, have been shown to be feasible and acceptable for targeting ASD-related functioning (e.g. Sandgreen et al., 2020; Valentine et al., 2020).

Two main types of digital technology were used in the reviewed treatment studies: telemedicine and ESM. Telemedicine interventions provided preliminary evidence of efficacy, feasibility, and acceptability for targeting transdiagnostic anxiety in ASD. These results must be interpreted with caution though as the usability of technology was impactful or not recorded, participant retention was variable, and only Conaughton et al. (2017) were not rated weak and used multi-modal and multi-informant measures of anxiety that are more likely to produce valid results in autistic samples. The only technology-based interventions that produced null results were ESM studies that targeted anxiety via a learning demonstration, specifically using visual media (i.e. photos and videos) to display peer modelling and social scripts of medical visits (i.e. Isong et al., 2014; Johnson et al., 2014). However, this pattern in findings ought to be interpreted with caution, chiefly because one of the two studies was minimally informative due to fundamental methodological limitations and found peer modelling to reduce anxiety when displayed via video goggles as opposed to via a DVD (i.e. Isong et al., 2014), but also in light of the limitations common to all studies discussed subsequently. Additionally, although extensive evidence shows that the situational factors inherent to medical visits do evoke anxiety that the peer modelling and social scripts attempted to address, such as novel social situations, unfamiliar procedures, and unclear rules/expectations (e.g. Bearss et al., 2016; Robertson et al., 2018), only two small-scale studies have provided evidence for the use of social scripts as a means of addressing this and they also only focused on medical visits (Drake et al., 2012; Johnson \& Bree, 2014). Further, there is a lack of convincing evidence for the use of such methods for improving social skills and behaviour in ASD (Kokina \& Kern, 2010; Leaf et al., 2020). Therefore, it is undeterminable whether these null results are attributable to the technology used, the interventions themselves, the presence of ASD, or the methodologies employed.
All 3 studies that used ESM for targeting anxiety during medical visits were weak in quality which thus diminishes the certainty of efficacy findings. On the other hand, the reported feasibility indicators were promising, and interventions across methodologies that involved ESM consistently reduced anxiety in most participants. Studies that used VRCBT for treating specific fears/phobias (i.e. Maskey et al., 2014, 2019a, 2019b, 2019c), specifically using VR for exposure and relaxation tasks, demonstrated the highest feasibility and acceptability, and all were moderate-to-strong in quality. In the non-ASD literature, the effectiveness of using VR for treating anxiety disorders in this way is the most established finding (Freeman et al., 2017). However, while all ESM studies used multimodal (e.g. self-report and behavioural) and multi-informant (e.g. child and parental) measures of anxiety, none of the VRCBT studies used physiological measures. Future studies may consider measuring physiological responses because, unlike the subjective measures used, their validity cannot be compromised by the ASD-specific challenges in atypical behavioural presentations of anxiety (Magiati et al., 2017) nor impairments in emotional literacy and interoceptive abilities (Kinnaird et al., 2019; Nicholson et al., 2019; Palser et al., 2018). This may explain why Maskey, Rodgers, Grahame et al. $2019 \mathrm{~b}$ found mixed findings across the subjective measures used. Furthermore, a recent meta-analysis indicates that physiological process measures better predict exposure therapy outcomes than those that are non-physiological (Rupp et al., 2017). However, as signposted by McVey (2019), the physiological markers of anxiety in ASD first need explicating to aid interpretability.

Future treatment studies should improve on blinding methods, use adequately powered RCTs with active comparators, include longer follow-ups (i.e. $>3$ months), and monitor concurrent psychopharmacological treatment regimes. Lastly, it must be considered that in the only two treatment studies that tested technology and therapistassisted CBT using an RCT design (i.e. Conaughton et al., 2017 and Maskey, et al., 2019b), improvements did not translate into loss of the targeted anxiety diagnosis for around $62 \%$ of participants. On the other hand, at least in neurotypical adults, remission rates for CBT vary and are moderated by the anxiety disorder diagnosis, and the number of sessions in both studies was lower than the 11 to 19 sessions recently deemed necessary for at least $50 \%$ of individuals to show clinically significant improvement (Levy et al., 2020). Furthermore, in autistic children (i.e. aged under 18 years) who were the common demographic across studies, long-term CBT interventions for anxiety are especially crucial and variability in outcomes may be attributable to the presence of atypical anxiety symptoms (Perihan et al., 2020). Future studies ought to take these factors into account where possible. 


\section{Overcoming Common Limitations of Reviewed Studies}

To bypass the common limitations of the reviewed assessment and treatment studies, future research ought to consider the influence of gender, levels of anxiety, ASD symptomatology, ASD and anxiety diagnoses, and comorbidities, as well as the use of behavioural measures of anxiety that capture atypical presentations. Furthermore, the generalisability of findings to the ASD population is questionable due to samples being self-selected and almost invariably consisting of young people with at least average verbal intelligence, no reported learning disabilities nor severe or complex mental health conditions.

\section{Strengths and Limitations of Review Methodology}

A strength of the search strategy was that it was informed by guidance from Salvador-Oliván et al. (2019) and McGowan et al. (2016), although due to resource constraints the eligibility criteria was restrictive (see Online Resources 3 and 4) and potentially afflicted by publication bias so it may not have fully captured relevant studies. There was also a risk of bias at data extraction since authors were not contacted to provide missing or additional data and since only one author undertook data extraction-though the completed data extraction form was checked by another author. Additionally, the common rubric used for the feasibility and efficacy indicators during synthesis must be interpreted with caution due to the variation in the indicators and definitions used across studies. Bias may have further been introduced when studies were grouped for synthesis as this was decided post-screening due to the wide range potential of assessments/treatments, technologies and anxiety disorders, and pairings therein. Nonetheless, another strength of the review was the approach to understanding discrepancies and uncertainties in the results which entailed systematically revisiting all elements of the final data extraction form so as to notice any patterns or differences in methodologies employed that may have explanatory power and to identify any research gaps that may provide a fuller understanding. Additionally, inter-rater reliability was desirable at study selection and quality assessment. Moreover, this review is the first of its kind and fulfilled its objectives of synthesising current evidence, alongside identifying the technologies used, anxiety targeted, and future research avenues.

\section{Conclusion}

Due to the lack of available studies, methodological heterogeneity, low quality, and small sample sizes, strong conclusions cannot be drawn. Nevertheless, this is to be expected of a research field in its infancy, and the narrative synthesis has broadly indicated that technology-aided assessments and treatments may be feasible and effective at targeting anxiety in ASD. The field would benefit from studies with universal feasibility indicators and outcome variables, adequate power, improved blinding procedures, and more representative samples. Future research should employ multi-modal and multi-informant measures of anxiety and consider the influence of gender, levels of anxiety, ASD symptomatology, and comorbidities. A notable research avenue is the direct testing of VR for inducing psychophysiological symptoms of anxiety, which could aid our conceptualisation of anxiety that findings indicate is distinct in ASD and elucidate the treatment mechanisms of VR exposure therapy.

Supplementary Information The online version contains supplementary material available at https://doi.org/10.1007/s40489-021-00275-6.

Acknowledgements The authors would like to thank $\mathrm{PhD}$ students Emilie Wildman and Heather McDonald for independently contributing to reliability checks at study inclusion level and quality assessment, respectively. The authors would also like to thank assistant clinical psychologists Aylana Brewster and Maisie Krisson for assessing the accuracy of the study description tables and Aylana Brewster for contributing to the reliability check for the additional study identified in the updated search. Lastly, the authors would like to thank Jennifer Watson (MSc student) for reviewing the PRISMA and SWiM reporting checklists. The authors acknowledge financial support from the Psychiatry Research Trust (PRT), the National Institute for Health Research (NIHR) Biomedical Research Centre for Mental Health at South London and Maudsley NHS Foundation Trust, and King's College London. The views expressed are those of the authors and not necessarily those of the PRT, NHS, the NIHR or the Department of Health.

Author Contribution LA formulated the review protocol; ran the search procedures, study screening, data extraction, quality assessment, reliability checks, and analysis; and wrote the manuscript. LV and ES oversaw the conception and procedures of the study. LV further contributed to reliability checks at study inclusion level and checking the accuracy of the data extraction forms. Disagreements in study inclusion, data extraction, and quality assessment were resolved by LV or ES. All authors were involved in drafting the manuscript and read and approved the final manuscript.

Funding LA was supported by a $\mathrm{PhD}$ studentship from the Psychiatry Research Trust (Grant reference: 0c Valmaggia). LV and ES acknowledge financial support from the National Institute for Health Research (NIHR) Biomedical Research Centre for Mental Health at South London and Maudsley NHS Foundation Trust and King's College London. The views expressed are those of the authors and not necessarily those of the PRT, NHS, the NIHR, or the Department of Health. 


\section{Declarations}

Ethics Approval Research Involving Human and Animal Participants.

This article does not contain any studies with human participants or animals performed by any of the authors.

Conflict of Interest The authors declare no competing interests.

Open Access This article is licensed under a Creative Commons Attribution 4.0 International License, which permits use, sharing, adaptation, distribution and reproduction in any medium or format, as long as you give appropriate credit to the original author(s) and the source, provide a link to the Creative Commons licence, and indicate if changes were made. The images or other third party material in this article are included in the article's Creative Commons licence, unless indicated otherwise in a credit line to the material. If material is not included in the article's Creative Commons licence and your intended use is not permitted by statutory regulation or exceeds the permitted use, you will need to obtain permission directly from the copyright holder. To view a copy of this licence, visit http://creativecommons.org/licenses/by/4.0/.

\section{References}

Altman, D. G. (1991). Statistics in medical journals: Developments in the 1980s. Statistics in Medicine, 10(12), 1897-1913. https:// doi.org/10.1002/sim.4780101206

American Psychiatric Association. (2013). Diagnostic and statistical manual of mental disorders (5th ed.). Washington, DC: Author.

Attwood, T. (2006). Asperger's syndrome and problems related to stress. In M. G. Baron, J. Groeden, G. Groeden, \& L. P. Lipsitt (Eds.), Stress and coping in autism (pp. 351-370). New York: Oxford University Press. https://doi.org/10.1093/med:psych/ 9780195182262.003.0014

Bearss, K., Taylor, C. A., Aman, M. G., Whittemore, R., Lecavalier, L., Miller, J., et al. (2016). Using qualitative methods to guide scale development for anxiety in youth with autism spectrum disorder. Autism, 20(6), 663-672. https://doi.org/10.1177/13623 61315601012

Bordignon, S., Endres, R. G., Trentini, C. M., \& Bosa, C. A. (2015). Memory in children and adolescents with autism spectrum disorder: A systematic literature review. Psychology and Neuroscience, 8(2), 211-245. https://doi.org/10.1037/h0101059

Campbell, M., McKenzie, J. E., Sowden, A., Katikireddi, S. V, Brennan, S. E., Ellis, S., et al. (2020). Synthesis without meta-analysis (SWiM) in systematic reviews: reporting guideline. $B M J$, 368(I6890). https://doi.org/10.1136/bmj.16890

Centre for Reviews and Dissemination. (2008). Core principles and methods for conducting a systematic review of health interventions. Systematic Reviews: CRD's guidance for undertaking reviews in health care (pp. 1-108). CRD, Univesity of York.

Chen, Y. W., Bundy, A., Cordier, R., Chien, Y. L., \& Einfeld, S. (2016). The experience of social participation in everyday contexts among individuals with autism spectrum disorders: An experience sampling study. Journal of Autism and Developmental Disorders, 46, 1403-1414. https://doi.org/10.1007/s10803-015-2682-4

Cieślik, B., Mazurek, J., Rutkowski, S., Kiper, P., Turolla, A., \& Szczepańska-Gieracha, J. (2020). Virtual reality in psychiatric disorders: A systematic review of reviews. Complementary Therapies in Medicine, 52, 102480. https://doi.org/10.1016/j. ctim.2020.102480

Conaughton, R. J., Donovan, C. L., \& March, S. (2017). Efficacy of an internet-based CBT program for children with comorbid high functioning autism spectrum disorder and anxiety: A randomised controlled trial. Journal of Affective Disorders, 218, 260-268. https://doi.org/10.1016/j.jad.2017.04.032

Diemer, J., Mühlberger, A., Pauli, P., \& Zwanzger, P. (2014). Virtual reality exposure in anxiety disorders: Impact on psychophysiological reactivity. World Journal of Biological Psychiatry, 15(6), 427-442. https://doi.org/10.3109/15622975.2014.892632

Drake, J., Johnson, N., Stoneck, A. V, Martinez, D. M., \& Massey, M. (2012). Evaluation of a coping kit for children with challenging behaviors in a pediatric hospital. Pedriatric Nursing, 38(4), 215-21. https://pubmed.ncbi.nlm.nih.gov/22970487/. Accessed 27 July 2020

Evans, D. W., Canavera, K., Kleinpeter, F. L., Maccubbin, E., \& Taga, K. (2005). The fears, phobias and anxieties of children with autism spectrum disorders and Down syndrome: Comparisons with developmentally and chronologically age matched children. Child Psychiatry Human Development, 36, 3-26. https://doi.org/10.1007/s10578-004-3619-x

Firth, J., Torous, J., Carney, R., Newby, J., Cosco, T. D., Christensen, H., \& Sarris, J. (2018). Digital technologies in the treatment of anxiety: Recent innovations and future directions. Current Psychiatry Reports, 20(6), 44. https://doi.org/10.1007/ s11920-018-0910-2

Fleiss, J. L., \& Cohen, J. (1973). The equivalence of weighted kappa and the intraclass correlation coefficient as measures of reliability. Educational and Psychological Measurement, 33(3), 613-619. https://doi.org/10.1177/001316447303300309

Fleiss, J. L., Levin, B., \& Paik, M. C. (2003). Statistical methods for rates and proportions (3rd ed.). Wiley.

Freeman, D., Reeve, S., Robinson, A., Ehlers, A., Clark, D., Spanlang, B., \& Slater, M. (2017). Virtual reality in the assessment, understanding, and treatment of mental health disorders. Psychological Medicine, 47(14), 2393-2400. https://doi.org/10.1017/S0033 $29171700040 X$

Gaigg, S. B., Flaxman, P. E., Mclaven, G., Shah, R., Bowler, D. M., Meyer, B., et al. (2020). Self-guided mindfulness and cognitive behavioural practices reduce anxiety in autistic adults: A pilot 8-month waitlist-controlled trial of widely available online tools. Autism, 24(4), 867-883. https://doi.org/10.1177/1362361320 909184

Gigante, M. A. (1993). Virtual reality: Definitions, history and applications. In R. A. Earnshaw, M. A. Gigante, \& H. Jones (Eds.), Virtual Reality Systems (pp. 3-14). Academic Press Limited.

Goodall, C. (2018). "I felt closed in and like I couldn't breathe": A qualitative study exploring the mainstream educational experiences of autistic young people. Autism \& Developmental Language Impairments, 3, 1-16. https://doi.org/10.1177/2396941518 804407

Grist, R., Croker, A., Denne, M., \& Stallard, P. (2019). Technology delivered interventions for depression and anxiety in children and adolescents: A systematic review and meta-analysis. Clinical Child and Family Psychology Review, 22, 147-171. https:// doi.org/10.1007/s10567-018-0271-8

Gujjar, K. R., van Wijk, A., Kumar, R., \& de Jongh, A. (2019). Are technology-based interventions effective in reducing dental anxiety in children and adults? A systematic review. Journal of Evidence-Based Dental Practice, 19(2), 140-155. https://doi.org/10. 1016/j.jebdp.2019.01.009

Hare, D. J., \& Chen, Y.-W. (2019). Experience sampling in the study of autism spectrum disorders. In J. Palmier-Claus, G. Haddon, \& F. Varese (Eds.), Experience Sampling in Mental Health Research (pp. 53-66). Routledge.

Hare, D. J., Gracey, C., \& Wood, C. (2016). Anxiety in high-functioning autism: A pilot study of experience sampling using a mobile platform. Autism, 20(6), 730-743. https://doi.org/10.1177/13623 61315604817 
Hare, D. J., Wood, C., Wastell, S., \& Skirrow, P. (2015). Anxiety in Asperger's syndrome: Assessment in real time. Autism, 19(5), 542-552. https://doi.org/10.1177/1362361314531340

Hektner, J. M., Schmidt, J. A., \& Csikszentmihalyi, M. (2007). Experience sampling method: Measuring the quality of everyday life. Thousand Oaks, California 91320: SAGE Publications Inc.

Hepburn, S., Blakeley-Smith, A., Wolff, B., \& Reaven, J. (2016). Telehealth delivery of cognitive-behavioral intervention to youth with autism spectrum disorder and anxiety: A pilot study. Autism, 20(2), 207-218. https://doi.org/10.1177/1362361315575164

Hollis, C., Sampson, S., Simons, L., Davies, E. B., Churchill, R., Betton, V., et al. (2018). Identifying research priorities for digital technology in mental health care: Results of the James Lind Alliance Priority Setting Partnership. The Lancet Psychiatry, 5(10), 845-854. https://doi.org/10.1016/S2215-0366(18)30296-7

Hollocks, M. J., Howlin, P., Papadopoulos, A. S., Khondoker, M., \& Simonoff, E. (2014). Differences in HPA-axis and heart rate responsiveness to psychosocial stress in children with autism spectrum disorders with and without co-morbid anxiety. Psychoneuroendocrinology, 46, 32-45. https://doi.org/10.1016/j.psyne uen.2014.04.004

Hossain, M. M., Khan, N., Sultana, A., Ma, P., McKyer, E. L. J., Ahmed, H. U., \& Purohit, N. (2020). Prevalence of comorbid psychiatric disorders among people with autism spectrum disorder: An umbrella review of systematic reviews and meta-analyses. Psychiatry Research, 287, 112922. https://doi.org/10.1016/j.psych res.2020.112922

Hu, M. X., Lamers, F., De Geus, E. J. C., \& Penninx, B. W. J. H. (2016). Differential autonomic nervous system reactivity in depression and anxiety during stress depending on type of stressor. Psychosomatic Medicine, 78(5), 562-572. https://doi.org/10.1097/ PSY.0000000000000313

Isong, I. A., Rao, S. R., Holifield, C., Iannuzzi, D., Hanson, E., Ware, J., et al. (2014). Addressing dental fear in children with autism spectrum disorders: A randomized controlled pilot study using electronic screen media. Clinical Pediatrics, 53(3), 230-237. https://doi.org/10.1177/0009922813517169

Johnson, N., Bree, O., Lalley, E. E., Rettler, K., Grande, P., Gani, M. O., \& Ahamed, S. I. (2014). Effect of a social script iPad application for children with autism going to imaging. Journal of Pediatric Nursing, 29(6), 651-659. https://doi.org/10.1016/j. pedn.2014.04.007

Johnson, N. L., \& Bree, O. A. (2014). Social script iPad application versus usual care before undergoing medical imaging: Two case studies of children with autism. Journal of Radiology Nursing, 33(3), 121-126. https://doi.org/10.1016/j.jradnu.2014.04.001

Kenny, L., Hattersley, C., Molins, B., Buckley, C., Povey, C., \& Pellicano, E. (2016). Which terms should be used to describe autism? Perspectives from the UK autism community. Autism, 20(4), 442462. https://doi.org/10.1177/1362361315588200

Kinnaird, E., Stewart, C., \& Tchanturia, K. (2019). Investigating alexithymia in autism: A systematic review and meta-analysis. European Psychiatry, 55, 80-89. https://doi.org/10.1016/j.eurpsy.2018. 09.004

Knutsen, J., Wolfe, A., Burke, B. L., Hepburn, S., Lindgren, S., \& Coury, D. (2016). A systematic review of telemedicine in autism spectrum disorders. Review Journal of Autism and Developmental Disorders, 3(4), 330-344. https://doi.org/10.1007/ s40489-016-0086-9

Kokina, A., \& Kern, L. (2010). Social storyTM interventions for students with autism spectrum disorders: A meta-analysis. Journal of Autism and Developmental Disorders, 40, 812-826. https://doi. org/10.1007/s10803-009-0931-0

Koumpouros, Y., \& Kafazis, T. (2019). Wearables and mobile technologies in autism spectrum disorder interventions: A systematic literature review. Research in Autism Spectrum Disorders, 66, 101405. https://doi.org/10.1016/j.rasd.2019.05.005

Lai, C. L. E., Lau, Z., Lui, S. S. Y., Lok, E., Tam, V., Chan, Q., et al. (2017). Meta-analysis of neuropsychological measures of executive functioning in children and adolescents with high-functioning autism spectrum disorder. Autism Research, 10(5), 911-939. https://doi.org/10.1002/aur.1723

Lake, J. K., Perry, A., \& Lunsky, Y. (2014). Mental health services for individuals with high functioning autism spectrum disorder. Autism Research and Treatment, 2014, 1-9. https://doi.org/10. 1155/2014/502420

Lau, Y., Leong, R., Uljarevic, M., Lerh, J. W., Rodgers, J., Hollocks, M. J., et al. (2020). Anxiety in young people with autism spectrum disorder: Common and autism-related anxiety experiences and their associations with individual characteristics. Autism, 24(5), 1111-1126. https://doi.org/10.1177/1362361319886246

Leaf, J. B., Ferguson, J. L., Cihon, J. H., Milne, C. M., Leaf, R., \& McEachin, J. (2020). A critical review of social narratives. Journal of Developmental and Physical Disabilities, 32, 241-256. https://doi.org/10.1007/s10882-019-09692-2

Levy, H. C., Worden, B. L., Davies, C. D., Stevens, K., Katz, B. W., Mammo, L., et al. (2020). The dose-response curve in cognitivebehavioral therapy for anxiety disorders. Cognitive Behaviour Therapy, 49(6), 439-454. https://doi.org/10.1080/16506073. 2020.1771413

Liu, C., Conn, K., Sarkar, N., \& Stone, W. (2008). Physiology-based affect recognition for computer-assisted intervention of children with autism spectrum disorder. International Journal of HumanComputer Studies, 66(9), 662-677. https://doi.org/10.1016/j.ijhsc. 2008.04.003

Macneil, B. M., Lopes, V. A., \& Minnes, P. M. (2009). Anxiety in children and adolescents with autism spectrum disorders. Research in Autism Spectrum Disorders, 3(1), 1-21. https://doi.org/10.1016/j. rasd.2008.06.001

Magiati, I., Ozsivadjian, A., \& Kerns, C. M. (2017). Phenomenology and presentation of anxiety in autism spectrum disorder. In \& J. J. W. C. M. Kerns, P. Renno, E. A. Storch, P. C. Kendall (Ed.), Anxiety in children and adolescents with autism spectrum disorder: Evidence-based assessment and treatment (pp. 33-54). Elsevier Academic Press. https://doi.org/10.1016/B978-0-12-805122-1. 00003-X

Marini, A., Ferretti, F., Chiera, A., Magni, R., Adornetti, I., Nicchiarelli, S., et al. (2018). Episodic future thinking and narrative discourse generation in children with Autism Spectrum Disorders. Journal of Neurolinguistics, 49, 178-188. https://doi.org/ 10.1016/j.jneuroling.2018.07.003

Maskey, M., Lowry, J., Rodgers, J., McConachie, H., \& Parr, J. R. (2014). Reducing specific phobia/fear in young people with autism spectrum disorders (ASDs) through a virtual reality environment intervention. PLOS ONE, 9(7), e100374. https://doi.org/ 10.1371/journal.pone.0100374

Maskey, M., McConachie, H., Rodgers, J., Grahame, V., Maxwell, J., Tavernor, L., \& Parr, J. R. (2019a). An intervention for fears and phobias in young people with autism spectrum disorders using flat screen computer-delivered virtual reality and cognitive behaviour therapy. Research in Autism Spectrum Disorders, 59, 58-67. https://doi.org/10.1016/J.RASD.2018.11.005

Maskey, M., Rodgers, J., Grahame, V., Glod, M., Honey, E., Kinnear, J., et al. (2019b). A randomised controlled feasibility trial of immersive virtual reality treatment with cognitive behaviour therapy for specific phobias in young people with autism spectrum disorder. Journal of Autism and Developmental Disorders, 49, 1912-1927. https://doi.org/10.1007/s10803-018-3861-x

Maskey, M., Rodgers, J., Ingham, B., Freeston, M., Evans, G., Labus, M., \& Parr, J. R. (2019c). Using virtual reality environments to 
augment cognitive behavioral therapy for fears and phobias in autistic adults. Autism in Adulthood, 1(2), 134-145. https://doi. org/10.1089/aut.2018.0019

McGowan, J., Sampson, M., Salzwedel, D., Cogo, E., Foerster, V., \& Lefebvre, C. (2016). PRESS Peer Review of Electronic Search Strategies: 2015 Guideline Statement. Journal of Clinical Epidemiology, 75, 40-46. https://doi.org/10.1016/j.jclinepi.2016.01.021

McVey, A. J. (2019). The neurobiological presentation of anxiety in autism spectrum disorder: A systematic review. Autism Research, 12(3), 346-369. https://doi.org/10.1002/aur.2063

Mineo, B. A., Ziegler, W., Gill, S., \& Salkin, D. (2009). Engagement with electronic screen media among students with autism spectrum disorders. Journal of Autism and Developmental Disorders, 39, 172-187. https://doi.org/10.1007/s10803-008-0616-0

Moher, D., Liberati, A., Tetzlaff, J., \& Altman, D. G. (2009). Preferred Reporting Items for Systematic Reviews and Meta-Analyses: The PRISMA Statement. PLoS Medicine, 6(7), e1000097. https://doi. org/10.1136/bmj.b2535

Newbutt, N., Sung, C., Kuo, H.-J., Leahy, M. J., Lin, C.-C., \& Tong, B. (2016). Brief report: A pilot study of the use of a virtual reality headset in autism populations. Journal of Autism and Developmental Disorders, 46, 3166-3176. https://doi.org/10.1007/ s10803-016-2830-5

NICE. (2013). Autism spectrum disorder in under 19s: support and management. Clinical guideline [CG170]. NICE.

Nicholson, T., Williams, D., Carpenter, K., \& Kallitsounaki, A. (2019). Interoception is impaired in children, but not adults, with autism spectrum disorder. Journal of Autism and Developmental Disorders, 49, 3625-3637. https://doi.org/10.1007/ s10803-019-04079-w

Ozsivadjian, A., Knott, F., \& Magiati, I. (2012). Parent and child perspectives on the nature of anxiety in children and young people with autism spectrum disorders: A focus group study. Autism, 16(2), 107-121. https://doi.org/10.1177/1362361311431703

Palser, E. R., Palmer, C. E., Galvez-Pol, A., Hannah, R., Fotopoulou, A., \& Kilner, J. M. (2018). Alexithymia mediates the relationship between interoceptive sensibility and anxiety. PLOS ONE, 13(9), e0203212. https://doi.org/10.1371/journal.pone.0203212

Parsons, S., Mitchell, P., \& Leonard, A. (2004). The use and understanding of virtual environments by adolescents with autistic spectrum disorders. Journal of Autism and Developmental Disorders, 34, 449-466. https://doi.org/10.1023/B:JADD.0000037421. $98517.8 \mathrm{~d}$

Patriquin, M. A., Hartwig, E. M., Friedman, B. H., Porges, S. W., \& Scarpa, A. (2019). Autonomic response in autism spectrum disorder: Relationship to social and cognitive functioning. Biological Psychology, 145, 185-197. https://doi.org/10.1016/j.biopsycho. 2019.05.004

Perihan, C., Burke, M., Bowman-Perrott, L., Bicer, A., Gallup, J., Thompson, J., \& Sallese, M. (2020). Effects of cognitive behavioral therapy for reducing anxiety in children with high functioning ASD: A systematic review and meta-analysis. Journal of Autism and Developmental Disorders, 50, 1958-1972. https://doi.org/10. 1007/s10803-019-03949-7

Popay, J., Roberts, H., Sowden, A., Pettricrew, M., Arai, L., Rodgers, M., et al. (2006). Guidance on the Conduct of Narrative Synthesis in Systematic Reviews. University of Lancaster.

Rezae, M., McMeekin, D., Tan, T., Krishna, A., Lee, H., Falkmer, T. (2019). Public transport planning tool for users on the autism spectrum: From concept to prototype Disability and Rehabilitation. Assistive Technology 1-11.https://doi.org/10.1080/17483107. 2019.1646818

Robertson, A. E., Stanfield, A. C., Watt, J., Barry, F., Day, M., Cormack, M., \& Melville, C. (2018). The experience and impact of anxiety in autistic adults: A thematic analysis. Research in Autism
Spectrum Disorders, 46, 8-18. https://doi.org/10.1016/J.RASD. 2017.11.006

Rupp, C., Doebler, P., Ehring, T., \& Vossbeck-Elsebusch, A. N. (2017). Emotional processing theory put to test: A meta-analysis on the association between process and outcome measures in exposure therapy. Clinical Psychology and Psychotherapy, 24(3), 697-711. https://doi.org/10.1002/cpp.2039

Rydzewska, E. (2016). Unexpected changes of itinerary - adaptive functioning difficulties in daily transitions for adults with autism spectrum disorder. European Journal of Special Needs Education, 31(3), 330-343. https://doi.org/10.1080/08856257.2016.1187889

Salvador-Oliván, J. A., Marco-Cuenca, G., \& Arquero-Avilés, R. (2019). Errors in search strategies used in systematic reviews and their effects on information retrieval. Journal of the Medical Library Association, 107(2), 210-221. https://doi.org/10.5195/ jmla.2019.567

Sandgreen, H., Frederiksen, L. H., Bilenberg, N. (2020). Digital interventions for autism spectrum disorder: A meta-analysis. Journal of Autism and Developmental Disorders 1-15.https://doi.org/10. 1007/s10803-020-04778-9

Santangelo, P. S., Ebner-Priemer, U. W., \& Trull, T. J. (2013). Experience sampling methods in clinical psychology. In J. S. Comer \& P. C. Kendall (Eds.), Oxford library of psychology. The Oxford handbook of research strategies for clinical psychology (pp. 188-210). Oxford, England: Oxford University Press.

Shane, H. C., \& Albert, P. D. (2008). Electronic screen media for persons with autism spectrum disorders: Results of a survey. Journal of Autism and Developmental Disorders, 38, 1499-1508. https:// doi.org/10.1007/s10803-007-0527-5

Suresh, L., \& George, C. (2019). Virtual reality distraction on dental anxiety and behavior in children with autism spectrum disorder. Journal of International Dental and Medical Research, 12(3), 1004-1010.

Taj-Eldin, M., Ryan, C., \& O’flynn, B., \& Galvin, P. . (2018). A review of wearable solutions for physiological and emotional monitoring for use by people with autism spectrum disorder and their caregivers. Sensors, 18(12), 4271. https://doi.org/10.3390/s18124271

Valentine, A. Z., Brown, B. J., Groom, M. J., Young, E., Hollis, C., \& Hall, C. L. (2020). A systematic review evaluating the implementation of technologies to assess, monitor and treat neurodevelopmental disorders: A map of the current evidence. Clinical Psychology Review, 80, 101870. https://doi.org/10.1016/j.cpr. 2020.101870

Vogan, V., Lake, J. K., Tint, A., Weiss, J. A., \& Lunsky, Y. (2017). Tracking health care service use and the experiences of adults with autism spectrum disorder without intellectual disability: A longitudinal study of service rates, barriers and satisfaction. Disability and Health Journal, 10(2), 264-270. https://doi.org/10. 1016/j.dhjo.2016.11.002

Wallace, S., Parr, J., \& Hardy, A. (2013). One in a Hundred: Putting families at the heart of autism research. Autistica.

Walz, L. C., Nauta, M. H., \& aan het Rot, M. . (2014). Experience sampling and ecological momentary assessment for studying the daily lives of patients with anxiety disorders: A systematic review. Journal of Anxiety Disorders, 28(8), 925-937. https://doi.org/10. 1016/j.janxdis.2014.09.022

World Health Organisation. (2009). Telemedicine: opportunities and developments in member states. Report on the second global survey on eHealth (Vol. 2). Geneva: World Health Organisation.

Publisher's Note Springer Nature remains neutral with regard to jurisdictional claims in published maps and institutional affiliations. 